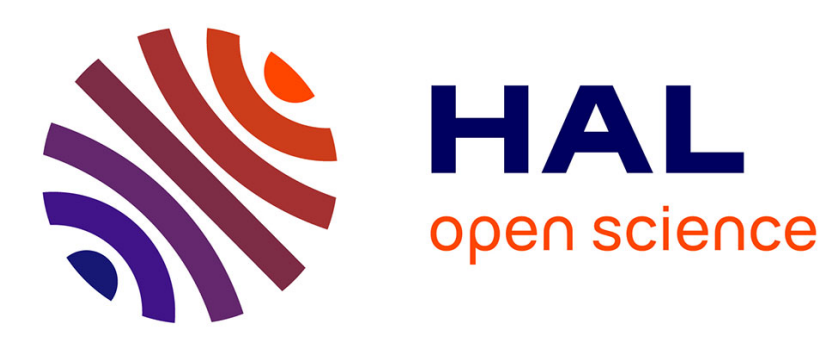

\title{
On a Simplified Model for Numerical Simulation of Wear During Dry Rolling Contacts
}

\author{
Luc Chevalier, A. Heddahak-Ouni, S. Cloupet
}

\section{To cite this version:}

Luc Chevalier, A. Heddahak-Ouni, S. Cloupet. On a Simplified Model for Numerical Simulation of Wear During Dry Rolling Contacts. Journal of Tribology, 2009, 131 (1), pp.1-15. 10.1115/1.3002322 . hal-00777136

\section{HAL Id: hal-00777136 \\ https://hal.science/hal-00777136}

Submitted on 23 Jan 2013

HAL is a multi-disciplinary open access archive for the deposit and dissemination of scientific research documents, whether they are published or not. The documents may come from teaching and research institutions in France or abroad, or from public or private research centers.
L'archive ouverte pluridisciplinaire HAL, est destinée au dépôt et à la diffusion de documents scientifiques de niveau recherche, publiés ou non, émanant des établissements d'enseignement et de recherche français ou étrangers, des laboratoires publics ou privés. 


\title{
On a Simplified Model for Numerical Simulation of Wear during Rolling Contacts
}

\author{
L. Chevalier ${ }^{1 *}$, A. Eddhahak-Ouni ${ }^{1}$, S. Cloupet $^{2}$ \\ ${ }^{1}$ LaM - Marne la Vallée University \\ 5 bd Descartes, Champs sur Marne \\ 77454 Marne la Vallée Cedex 2 \\ ${ }^{2}$ INRETS LTN \\ 2, Av. du G ${ }^{\text {al }}$ Malleret-Joinville, \\ 94114 ARCUEIL Cedex, France \\ * corresponding author \\ Tel: 0160957785 \\ Fax: 0160957799 \\ Email: luc.chevalier@univ-mlv.fr
}

\begin{abstract}
We deal with rolling contact between quasi-identical bodies. As normal and tangential problems are uncoupled in that case, the simplified approach to determine contact area and normal loading distribution for rolling contact problems is presented in a first part. In the second part, the solution of the tangential problem is used to reactualised the rolling profiles and enables to follow the wear evolution versus time.

The method used to solve the normal problem is called Semi Hertzian Approach with Diffusion (SHAD for short). It allows fast determination of the contact area for non Hertzian cases. The method is based on geometrical indentation of bodies in contact: The contact area is found with correct dimensions but affected by some irregularities coming from the curvature's discontinuity that may arise during a wear process. Diffusion between the independent stripes smoothes the contact area and pressure distribution.

The tangential problem is also solved on each stripe of the contact area using an extension of the simplified approach developed by Kalker and called Fastsim. At the end, this approach gives the dissipated power distribution in the contact during rolling and this power is related to wear by the Archard's law.

This enables to reactualise the profiles of the bodies in contact and to follow the geometrical evolution due to the wear phenomena.
\end{abstract}

Keywords: Rolling contact; Semi Hertzian method; conforming contact; wear simulation. 


\section{Introduction}

Wear evolution during contact in industrial machinery (Rollers on cam in blow molding machine for example [1]) is due to many factors: contact geometry, normal load, machine structure, internal friction in components, material rigidity and hardness, geometrical defects... In this industrial context, several parameters are not precisely known. In a previous paper, Chevalier and al. [2] presented a probabilistic approach which requires numerous calculations to describe random evolution of uncertain parameters. Number of calculation increases even more when non stationary processes are involved which is often the case in industrial machinery. In this context, we have developed a simplified method which allows fast simulation of wear evolution occurring in rolling contact under load.

When wear phenomenon occurs, the local geometry of bodies in contact may be modified because of the energy dissipated locally. Consequently, solids curvatures are no more constant and bodies in contact may become conforming. This consequence raises a problem since the Hertz theory which can be used for quick determination of the contact area and contact pressure calculation does not allow to solve the contact problem in the case of tangential loading with friction and non constant curvatures of the bodies in contact. For this general case, it has been established (see Jacobson \& Kalker [3] for example) that for two quasiidentical bodies (same elastic material properties: G shear modulus and $v$ Poisson's ratio), normal and tangential problems are uncoupled and relative displacements are related to tractions $\tau x, \tau y$ and pressure $\mathrm{p}$ by relations of Eq. 1 .

$$
\begin{aligned}
& u(x, y)=\frac{1}{\pi G} \iint_{\text {contact area }}\left(\left\{\frac{1-v}{r}+\frac{v\left(x-x^{\prime}\right)^{2}}{r^{3}}\right\} \tau_{x}\left(x^{\prime}, y^{\prime}\right)+\frac{v\left(x-x^{\prime}\right)\left(y-y^{\prime}\right)}{r^{3}} \tau_{y}\left(x^{\prime}, y^{\prime}\right)\right) d x^{\prime} d y^{\prime} \\
& v(x, y)=\frac{1}{\pi G} \iint_{\text {contact area }}\left(\frac{v\left(x-x^{\prime}\right)\left(y-y^{\prime}\right)}{r^{3}} \tau_{x}\left(x^{\prime}, y^{\prime}\right)+\left\{\frac{1-v}{r}+\frac{v\left(y-y^{\prime}\right)^{2}}{r^{3}}\right\} \tau_{y}\left(x^{\prime}, y^{\prime}\right)\right) d x^{\prime} d y^{\prime} \\
& w(x, y)=\frac{1-v}{\pi G} \iint_{\text {contact area }} \frac{p\left(x^{\prime}, y^{\prime}\right)}{r} d x^{\prime} d y^{\prime}
\end{aligned}
$$


Global $\mathrm{G}$ and $v$ are defined from the elastic coefficients for each body by:

$\frac{1}{G}=\frac{1}{2 G_{1}}+\frac{1}{2 G_{2}} \quad ; \quad \frac{v}{G}=\frac{v_{1}}{2 G_{1}}+\frac{v_{2}}{2 G_{2}}$

Where $(\mathrm{x}, \mathrm{y}, \mathrm{z})$ are the coordinates in $\mathrm{x}$-direction, $\mathrm{y}$-direction and $\mathrm{z}$-direction of point $\mathrm{M}$ and $\left(\mathrm{x}^{\prime}, \mathrm{y}^{\prime}, \mathrm{z}^{\prime}\right)$ the coordinates of point $\mathrm{M}^{\prime}$ as presented in Fig.1. In the case of constant curvature in the contact area, the third relation of Eq.1 is analogous to the Hertz problem. Pressure distribution $p(x, y)$ is elliptic, and contact area is an ellipse where half lengths in both $\mathrm{X}$ and $\mathrm{Y}$ directions are denoted $\mathrm{a}$ and $\mathrm{b}$ and can be solved separately from the first two relations of Eq.1. We will recall some results needed to detail the semi hertzian approach in the following. The tangential problem is still coupled with the normal problem by the friction law.

The simplified approach based on the bodies indentation has been presented in [4] in order to estimate the contact area during rolling contact under a normal load. This approximation is built on Hertz theory when solids curvatures are no longer constant but requires that curvatures must be positive. When this is no longer the case (i.e. solids with negative or null curvatures), this approach fails. We propose to solve this point by using a method based on the diffuse approximation [5]. This method called semi hertzian approach with diffusion (SHAD for short) allows to estimate the contact area with a good precision. SHAD can easily be extended in order to solve the tangential problem in the cases of steady or non steady rolling state. For example, one may use the Fastsim Algorithm [6] on the contact area discretised by independent stripes. This is presented in the second part and the traction and slip velocity computed help to determine the wear evolution. The Archard's law is adapted to the determination of the local depth increasing. At each step of the simulation, the local geometry is actualized and this process allows to follow the evolution of wear versus time including the modification of the local curvatures. 


\section{Normal problem: Semi Hertzian Approach with Diffusion}

\subsection{Semi Hertzian approach}

The Semi Hertzian approach aims to find the contact area between two revolution bodies with non constant curvatures in a normal contact. It is based on several results of the Hertz theory that we first recall.

\subsubsection{Some results of the Hertz theory}

We consider two revolution bodies numbered 1 and 2 under a normal load $F$. The radii of curvatures in the plane $(x O z)$ are $R_{l x}$ and $R_{2 x}$, those in the plane $(y O z)$ are $R_{l y}$ and $R_{2 y}$ (see Fig. 2). We denote by $E$ et $v$ respectively the Young's modulus and the Poisson's ratio of both bodies (quasi-identical case). In that particular case, Hertz showed that the contact area is elliptic and its semi axes lengths are given below:

$a=m\left(\frac{3 F}{2(A+B)} \frac{\left(1-v^{2}\right)}{E}\right)^{1 / 3} \quad b=n\left(\frac{3 F}{2(A+B)} \frac{\left(1-v^{2}\right)}{E}\right)^{1 / 3}$

where $A$ and $B$ are defined as follow:

$A=\frac{1}{2}\left(\frac{1}{R_{1 x}}+\frac{1}{R_{2 x}}\right), B=\frac{1}{2}\left(\frac{1}{R_{1 y}}+\frac{1}{R_{2 y}}\right)$

$m, n$ are coefficients resulting from elliptic integrals depending only on the curvatures $A$ and $B$. They can be interpolated by Eq.5.

$m=\frac{\alpha}{120}+\frac{1}{4}, n=47.62 \alpha^{-0.8465}, \alpha=\operatorname{acos} \frac{|A-B|}{A+B}$

The shape ratio of the elliptic contact area is given by Eq.6.

$k=\frac{b}{a}=\frac{n}{m}$ 
The pressure distribution and the elastic displacement between the curvatures far from the contact are respectively given by Eqs.7 and 8 .

$$
\begin{aligned}
& p(x, y)=\frac{3 F}{2 \pi a b} \sqrt{1-\frac{x^{2}}{a^{2}}-\frac{y^{2}}{b^{2}}} \\
& \delta=r\left(\frac{3 F}{2} \frac{\left(1-v^{2}\right)}{E}\right)^{2 / 3}(A+B)^{1 / 3}
\end{aligned}
$$

$r$ is also a coefficient defined by an integral like $m$ and $n$.

\subsubsection{Semi Hertzian method}

The proposed technique to estimate the contact area in the case of non constant curvatures in the plane $(y O z)$ is based on the indentation principle.

First, we impose an indentation $h_{0}$ between two rigid bodies with constant curvatures $A$ and $B$ near the contact zone (see Fig. 3). As a consequence, the geometric intersection is an ellipse defined by Eq.9.

$h_{0}=A x^{2}+B y^{2}$

The shape ratio of this ellipse is given by Eq.10.

$k^{\prime}=\frac{b^{\prime}}{a^{\prime}}=\sqrt{\frac{A}{B}}=\sqrt{\lambda}$

This ratio is different from the Hertz ellipse ratio of Eq. 6 because we do not take bodies local deformation into account. It is necessary to make a correction in order to get the same dimensions than the one obtained by the Hertz theory. Because we will study the cases of rolling contact where one of the solids must have an axisymmetry, we choose to correct only the curvature $A$ as follow:

$A \rightarrow A_{c}=k^{2} B$

Eqs. 9 and 11 allow us to calculate the semi axis $a$ as: 
$a=\sqrt{\frac{h_{0}}{A_{c}}}$

To have the same axis lengths as the Hertz ellipses, $h_{0}$ must to be equal to:

$h_{0}=a^{2} A_{c}=b^{2} B=\frac{n^{2}}{r} \frac{\delta}{1+\lambda}$

Based upon Eqs. 3 and 13, one can define the mean "rigidity" $K$ of the contact area as follow:

$$
\frac{K}{2 b}=\frac{F}{2 b h_{0}}=\frac{E}{3\left(1-v^{2}\right)} \frac{1+\lambda}{n^{3}}
$$

As it can be seen in Eq.14, the "rigidity" depends on global variables like $\lambda$ and $n$.

For non Hertzian bodies these variables do not make sense and we must to define this rigidity locally in every abscissa $y$ independently. We cut the potential contact area in stripes parallel to $x$ where $y_{i}$ is the center of the strip ' $\mathrm{i}$ '. We note by $\Delta y$ the width of the stripe (see Fig.4). The profile geometry of the roller is defined by the equation (y) and $z_{i}$ is the value at every abscissa $y_{i}$. Local curvature $B_{i}$ can be calculated as follow:

$B_{i}=\frac{\left(1+z_{i}^{\prime 2}\right)^{3 / 2}}{z_{i}^{\prime \prime}}$

where $z^{\prime}$ and $z$ ” are first and second derivatives of the profile equation $z(y)$. In the plane $(x O z)$, the roller is axisymmetric, thus the calculation of local curvature $A_{i}$ is easier.

For every stripe, the curvatures $A_{i}, B_{i}$ and the shape ratio $k_{i}$ are calculated and we proceed to the correction of $A_{i}$ as proposed in Eq.11. Then we are able to define local parameters of the virtual ellipse associated to stripe ' $\mathrm{i}$ ' for example its length $a_{i}$ in the $x$ direction by:

$a_{i}=\sqrt{\frac{h_{i}}{A_{c}}}$

where $h_{i}$ is the local distance between the bodies after indentation. This distance is given by Eq.17.

$h_{i}=h_{0}-z_{i}$ 
Using the relation of Eq.14, one can define the normal rigidity of the stripe ' $i$ ':

$$
K_{i}=\frac{E}{2\left(1-v^{2}\right)} \cdot \frac{1+\lambda_{i}}{n_{i}^{3}} \cdot \Delta y
$$

The normal rigidity of the central stripe is 1.5 times more important than the mean rigidity of the contact area. Then the stripe contribution in the global normal load $F$ is given by:

$$
\Delta N_{i}=K_{i} \cdot h_{i}
$$

The indentation $h_{0}$ is obtained when Eq.20 is satisfied.

$$
\sum_{i} \Delta N_{i}=F
$$

This simplified method is illustrated on a few examples in the following section.

\subsubsection{Results and discussion}

In this first example, the two solids 1 and 2 are respectively a roller and a plane path. Solid 1 has a revolution radius equal to $500 \mathrm{~mm}$, its profile in the plane $(y O z)$ presents a curvature discontinuity: as long as $-6 \mathrm{~mm}<y<6 \mathrm{~mm}$, the curvature $B$ is constant and equal to $0.5 \mathrm{~m}^{-1}$,

else the curvature is equal to zero (see Fig. 5). Elastic constants are: $E=2.110^{11} \mathrm{~Pa}$ and Poisson's ratio: $v=0.3$. Simplified approach is tested on this example with two normal loads in order to see the contact patch evolution. For $F=23 \mathrm{kN}$, we are in a quasi Hertzian case: the contact patch is elliptic and nearly coincides with the analytical solution of Hertz (Fig. 6). In fact, contact occurs in the zone with constant curvature and the width of the contact patch does not exceed $12 \mathrm{~mm}$. If normal load increases, the contact area will exceed the width of 12 $\mathrm{mm}$ and the contact will not be Hertzian any more. For $F=200 \mathrm{kN}$, the normal load is important, so the contact patch's width will exceed $12 \mathrm{~mm}$. Since $B$ is equal to zero outside the central zone of the profile of the roller, the Eq.10 used in semi hertzian method becomes meaningless. In order to respect the method conditions, we will slightly increase $B$ to nearly zero. In other words, we will introduce a very slight convexity to the profile of the roller while 
$y>6 \mathrm{~mm}$ and $y<-6 \mathrm{~mm}$. The contact area obtained by Semi Hertzian approach seems to have the same dimensions as that given by the exact theory (see Fig. 7) but we can notice the presence of discontinuities in both contact area and normal load distribution. These jumps occur at $y=-6 \mathrm{~mm}$ and $y=6 \mathrm{~mm}$ where roller curvature changes: as $B$ is discontinuous and by the correction of $A, A_{c}$ will be discontinuous as well (Eq. 11). Moreover, in the Semi Hertzian approach the local geometry $a_{i}$ is calculated locally and independently of the stripes ' $\mathrm{i}-1$ ' and ' $\mathrm{i}+1$ ' (Eq. 16), thus we can expect discontinuities in the contact area every time the curvature $\mathrm{B}$ is discontinuous.

\subsection{Semi Hertzian Approach with Diffusion (SHAD)}

Even if global contact area dimensions are correct we propose to introduce coupling between stripes into semi hertzian method in order to have a more realistic continuous contact patch. This method called also "diffuse approximation" presented in [5] and already used for contact path determination in [7] provides a nodal approximation to a function simply known by a finite number of points. This process provides also the $M$ first successive derivatives of this function.

\subsubsection{Diffuse element method}

For our case, the parameter to be diffused is the curvature $B(y)$. We first estimate the Taylor expansion of $B$ at $y$ as follow:

$B(y)=\sum_{i=0, M} p_{1 i}(y) \cdot \alpha_{i}^{*}(y)$

$\alpha^{*}(y)$ is chosen to minimize quadratic expression given by:

$I_{y}(\alpha)=\sum_{j=1, P} w\left(y, y_{j}-y\right)\left[B\left(y_{j}\right)-p_{1}^{T}\left(y_{j}-y\right) \cdot \alpha(y)\right]^{2}$ 
Where $P$ represents the number of points used for discretization and $w$ is the weight function of exponential form given by:

$w(\widetilde{y})=\exp \left(-C \tilde{y}^{2}\right)$

$\tilde{y}$ is a characteristic dimension defined by:

$\tilde{y}=\frac{y}{L_{y}}$

where $L_{y}$ is a characteristic length equal to the width of the contact zone in the $y$ direction. The minimum of $I_{y}$ is reached at $\alpha^{*}$ which satisfies the following system:

$A_{1}(y) \cdot \alpha(y)=b_{1}(y) \quad$ where $:\left\{\begin{array}{l}A_{1}(y)=\sum w\left(y, y_{j}-y\right) p_{1}\left(y_{j}-y\right) p_{1}{ }^{T}\left(y_{j}-y\right) \\ b_{1}(y)=\sum w\left(y, y_{j}-y\right) B\left(y_{j}\right) p_{1}\left(y_{j}-y\right)\end{array}\right.$

Once system of Eq. 25 is solved, the function initially discontinuous $B(y)$ will be given by:

$B(y)=\alpha_{0}(y)$ et $D^{q}(B(y))=q ! \alpha_{q}(y)$

where $q$ varies from 1 to $M$. Let's look back to the reference example above with a normal force of $200 \mathrm{kN}$. We note that initial curvature is maintained equal to zero in Zone II: owing to diffusion, B will increase and Semi Hertzian method continue to be applied.

It's of interest to mention that there are several choices for the coefficient $C$ defined in Eq.23. Each coefficient provides us a new system like Eq.25 to be solved and consequently a different diffused expression of $B(y)$. The questions which arise are: is there and what is the optimal coefficient $C^{*}$ ?

In order to answer this question, we managed different calculation with SHAD using different values of $\mathrm{C}$ and compared with one reference calculation made by using a finite element code. It allows to define an error as follow:

$\operatorname{Error}(C)=\operatorname{Error} 1+\operatorname{Error} 2=\left(\frac{\sum_{\text {stripes }}|d S-d S|}{S}\right)_{1}+\left(\frac{\sum_{\text {stripes }}|d N-d n|}{N}\right)_{2}$

where: 
- Error 1 and Error 2 are respectively the errors on the contact area and the distribution of the normal load by unit length.

- $d S$ and $d s$ are respectively stripes areas obtained by the exact approach and SHAD.

- $d N$ et $d n$ are respectively the stripes areas of the normal load by length obtained by the exact approach and SHAD.

- $S$ and $N$ are respectively the total area of the contact area and normal load by length given by reference solution.

The optimal diffusion coefficient $C^{*}$ is the one that minimizes the error given above: it satisfies a good compromise between contact area and pressure distribution. In that case $C^{*}$ is equal to 3.5 , we note that error resulting is nearly $7 \%$ (Fig. 8). In order to test the sensibility of the optimal coefficient $C^{*}$ to the problem characteristics, calculations have been fulfilled by SHAD to study the influence of the normal load and the curvature in the plane $(x O z)$ on contact area and pressure distribution. The results are shown in Fig. 9. This graph shows the variation of $C^{*}$ when the normal load varies, the curvature $A$ is unchanged and maintained equal to $1 \mathrm{~m}^{-1}$. When $F$ is superior to $40 \mathrm{kN}, C^{*}$ doesn't vary a lot, it belongs to [3.6; 6]. Furthermore, when $F$ is superior or equal to $120 \mathrm{kN}$, we can notice the presence of a landing in which this coefficient is constant: it's always equal to 3.5. We have noticed that if we consider a mean value of $C^{*}$ for all the loads superior to $40 \mathrm{kN}$ the resulting error increases by almost $0.1 \%$. On the other hand, we notice that when $F$ is smaller than $40 \mathrm{kN}, C^{*}$ increases and reaches 11 at $30 \mathrm{kN}$. Likewise calculations have been made with varied curvatures $A$, the normal effort is taken equal to $200 \mathrm{kN}$. The results are given below.

Fig. 9 illustrates the variation of $C^{*}$ when the curvature $A$ changes. We notice that $C^{*}$ is constant when $A$ is superior to $1.5 \mathrm{~m}^{-1}$, in other words when the radius of the roller in the plane $(x O z)$ is less than $334 \mathrm{~mm}$. Nevertheless when $1<A<1.5 C^{*}$ belongs to [2, 3.5] so 
error doesn't vary much when we use a mean single value for all the curvatures superior or equal to 1.5 .

Now when $A$ is less than $1 \mathrm{~m}^{-1}$, changes are more important and we can see that $C^{*}$ varies between 0 and 6 . As well as the previous case, we have tested a mean value for $C^{*}$ in this region and we noticed that only $0.5 \%$ has to be added to the resulting error. So, we demonstrated by theses calculations that a unique value of $\mathrm{C}^{*}$ around 4 gives an accurate estimation of the normal problem solution for a wide range of various loads and curvatures.

\subsubsection{Several critical cases of curvature $B$ values}

In this section, we will treat a particular case where $B$ is 0 . This situation arises a problem for Hertzian formulae require positive curvatures (see Eq.10). With SHAD, one can notice that once $B$ is diffused the curvature is not equal to zero any more and it is possible to apply safely the Semi Hertzian method. Thus during our application, we will maintain $B$ to zero so we avoid discussion about the optimal positive value of $B$ to be chosen when it's zero.

Let's consider the example of the contact roller/plane where geometric characteristics of the upper solid are defined as follow. In the plane $(x O z)$, the roller has the same geometric characteristics as in the previous paragraph. In the plane $(y O z)$, we distinguish a central zone corresponding to $y$ in $[-10 \mathrm{~mm}, 10 \mathrm{~mm}]$ in which the curvature is equal to zero, then when $y>$ $10 \mathrm{~mm}$ or $y<-10 \mathrm{~mm}$, the curvature $B$ becomes equal to $0.375 \mathrm{~m}^{-1}$ (see Fig. 10). The normal load $F$ is $200 \mathrm{kN}$. The diffuse element method gives $C^{*}=4$ with an error equal to $5 \%$. In Fig.11, we show both diffused and non diffused curvature. The curvature $B$ is maintained equal to zero in Zone I, the contact area and the distribution of the normal load by unit of length are also drawn.

Once again, SHAD shows excellent agreement with the exact approach results: contact areas is closely confused by both approaches as well as distribution of the normal load by unit of 
length, jumps at discontinuities zones are disappeared owing to diffusion (Fig. 11). In order to validate this simplified approach for general cases, calculations were made for different normal loads. Results are given Fig.12: the plot shows the variation of the optimal diffusion coefficient with normal load. One can notice that this coefficient is almost constant because it varies between 3 and 4 . Thus, provided an additional error of less than $1 \%$, we can for example consider a mean diffusion coefficient for all the loads.

When $B$ is negative, the correction of $A$ will lead to a negative $A_{c}$. Consequently, Eq.12 will no longer be valid and problem arises again on the using of Hertzian formulae. In order to avoid this problem, we simply suggest to put $B$ at zero when it's negative. Then diffusion guarantees that $B$ will be superior to zero. In this section, we present the example of the contact between a plane and a roller with negative curvature under a normal load equal to 200 $\mathrm{kN}$. Geometrical characteristics of the roller profile along $y$ will be altered. We suppose that $B$ is equal to $-0.25 \mathrm{~m}^{-1}$ when $y$ belongs to [-6 mm, $6 \mathrm{~mm}$ ] and $0.375 \mathrm{~m}^{-1}$ otherwise as it's shown in Fig.13. With SHAD the curvature $B$ is smoothed after discontinuity. This leads to a smooth contact area very close to the one given by the exact method. It's also the case for the normal load by length (Fig. 14). In this example, the optimal diffusion coefficient is equal to 5.5, only an error of $7 \%$ is considered. Like the previous case we studied the variation of $C^{*}$ for different normal loads: we can notice on Fig. 15 that $C^{*}$ is nearly the same for all cases. Its mean value is around 4.5 .

\section{Tangential problem and wear simulation}

In this section, we focus on the rolling contact analysis. We briefly recall the kinematics relations and friction laws needed to solve the tangential problem. Then we present the simplified approach extended from Fastsim and that we will use for the numerical developments. 


\subsection{Tangential problem}

In the case of two rigid bodies in contact at point $\mathrm{O}(x=0, y=0)$, the rigid sliding velocity $\overrightarrow{V_{r s}}$ is defined by system of Eq.28:

$\left\{\begin{array}{l}V_{r s x}=R_{1 x} \omega_{1}-R_{2 x} \omega_{2}+y \varphi_{x} \omega_{1} \\ V_{r s y}=-R_{1 x} \varphi_{z} \omega_{1}-x \varphi_{x} \omega_{1}\end{array}\right.$

Where $V_{r s x}$ and $V_{r s y}$ are the x-coordinate and y-coordinate of $\overrightarrow{V_{r s}}, \varphi_{x}$ and $\varphi_{z}$ are angular defects between the two bodies. Since bodies have an elastic behaviour, the relative velocity has a complementary term and system of Eq. 28 becomes:

$$
\left\{\begin{array}{l}
w_{x}=R_{1 x} \omega_{1}-R_{2 x} \omega_{2}+y \varphi_{x} \omega_{1}-V \frac{\partial u(x, y)}{\partial x} \\
w_{y}=-R_{1 x} \varphi_{z} \omega_{1}-x \varphi_{x} \omega_{1}-V \frac{\partial v(x, y)}{\partial x}
\end{array}\right.
$$

Where $\vec{w}(x, y)$ is the sliding velocity between two elastic bodies and $w_{x}$ and $w_{y}$ its components. $u(x, y)$ and $v(x, y)$ are the relative displacements in $\mathrm{x}$-direction and y-direction. $w$ can be divided by $V$ and system (3) becomes:

$$
\left\{\begin{array} { l } 
{ \frac { w _ { x } } { V } = v _ { x } - y \phi - \frac { \partial u ( x , y ) } { \partial x } } \\
{ \frac { w _ { y } } { V } = v _ { y } + x \phi - \frac { \partial v ( x , y ) } { \partial x } }
\end{array} \text { with } \left\{\begin{array}{l}
v_{x}=\frac{R_{1 x} \omega_{1}-R_{2 x} \omega_{2}}{V} \\
v_{y}=-\frac{R_{1 x} \varphi_{z} \omega_{1}}{V} \\
\phi=-\frac{\varphi_{x} \omega_{1}}{V}
\end{array}\right.\right.
$$

$v_{x}$ and $v_{y}$ are called longitudinal and lateral creepages ( $v_{x}$ is also usually called "slip rate"), $\phi$ is the spin in the contact area (Fig. 16) and $V$ is the speed of the theoretical contact point when bodies are supposed to be perfectly rigid. In our case, we want to know the tangential traction $\vec{\tau}(x, y)$, the normal load $p(x, y)$ and the sliding velocity $\vec{w}(x, y)$ in the contact area. This problem can be solved with two approaches: The exact theory at first and second the simplified theory. According to the Coulomb's friction laws (Eq.31), if sliding velocity is 
equal to zero then the tangential traction is lower than friction coefficient multiplied by normal pressure. Otherwise the tangential traction is equal to friction coefficient multiplied by normal pressure.

$$
\left\{\begin{array}{c}
\vec{w}(x, y)=\overrightarrow{0} \Rightarrow|\vec{\tau}(x, y)| \leq \mu p(x, y) \\
\vec{w}(x, y) \neq \overrightarrow{0} \Rightarrow|\vec{\tau}(x, y)|=\mu p(x, y) \quad \text { and } \vec{\tau}(x, y)=-\frac{\mu p(x, y)}{|\vec{w}(x, y)|} \vec{w}(x, y)
\end{array}\right.
$$

$u, v, w$ are related with $\tau_{x}, \tau_{y}$ and $p$ by the elastic behaviour laws of the two bodies as presented in Eq.1 for quasi-identical elastic bodies. In section 2 we have presented the SHAD method to solve the normal problem. Next we will present the simplified approach to solve the tangential one.

\subsubsection{Fastsim extension}

An alternative to the prohibitive CPU time needed by the initial approach is to use the Fastsim algorithm as developed by Kalker. Let's examine the proposed simplifications: the local stiffness is modelled as a linear relation between tangential traction and relative displacements $u$ and $v$ (i.e. $u=L . \tau_{x}$. and $v=L . \tau_{y}$ ). The $L$ value depends of $C_{i j}$ coefficients, shear elastic modulus $G$ and ellipse dimensions $a$ and $b$, but we must introduce three values to ensure that the total tangential force components $T_{x}$ and $T_{y}$ of Linear Kalker Theory [8] are identical in the case of global adhesion:

$$
L_{1}=\frac{8 a}{3 C_{11} G} \quad, L_{2}=\frac{8 a}{3 C_{22} G} \quad, L_{3}=\frac{\pi a \sqrt{a / b}}{4 C_{23} G}
$$

The simplified problem to solve is expressed by the relations of Eq.33. It is easy to see that for an elliptic pressure distribution, the slip component will lead to infinity at the trailing edge of the contact. This is in contradiction with Fig. 4 and one can solve this problem using a 
parabolic pressure distribution. This is an approximation that increases the maximum pressure $P_{o}$ for $25 \%$ but gives good agreement in the tangential problem case.

$$
\left\{\begin{array}{l}
\frac{w_{x}}{V L}=\frac{v_{x}}{L_{1}}-\frac{\phi y}{L_{3}}-\frac{\partial \tau_{x}}{\partial x} \\
\frac{w_{y}}{V L}=\frac{v_{y}}{L_{2}}+\frac{\phi x}{L_{3}}-\frac{\partial \tau_{y}}{\partial x}
\end{array}\right.
$$

$L$ is the mean flexibility obtained by weighting each flexibility $L_{i}(\mathrm{i}=1$ to 3 ) by the associated creepage value. No theoretical justification can be proposed for the definition of Eq.34.

$L=\frac{v_{x} L_{1}+v_{y} L_{2}+\sqrt{a b} \phi L_{3}}{\sqrt{v_{x}^{2}+v_{y}^{2}+a b \phi^{2}}}$

Solving the system of Eq. 33 leads to an analytical solution when spin is neglected.

\subsubsection{Comparison with the complete approach}

Fastsim algorithm solves the tangential problem in the case of Hertzian bodies . It's 100 times quicker than any algorithm used to solve the complete rolling contact problem. This was proved by comparison with the approached results obtained by Fastsim and a software that solves the exact theory like 'Contact' for example [9]. Consequently we will use the Fastsim approximation instead of the exact system in what follows.

Wear generated at each time step modifies the curvatures and generates discontinuities. The example of Fig. 17, is representative of the problem to solve during the wear simulation process. Plots of Fig.18, 19 and Fig. 20 clearly show the good correlation between the solution of 'Contact' and the SHAD \& Fastsim approaches as well for the contact path and pressure distribution than for slip velocity or tangential tractions distribution. 


\subsection{Wear simulation}

\subsubsection{Archard's wear model}

A model for material loss due to the cyclic rolling contact loading is provided by Archard's law [10]. A similar form was proposed by $\mathrm{Zi} \mathrm{Li} \mathrm{\&} \mathrm{Kalker} \mathrm{[11]} \mathrm{and} \mathrm{the} \mathrm{mathematical}$ expression is given by Eq.35.

$W=\frac{K}{H} T L$

$W\left(\mathrm{~m}^{3}\right)$ is the worn volume, $L(\mathrm{~m})$ is the sliding length of the abrasive particle, $T(\mathrm{~N})$ is the tangential load and $H$ $\left(\mathrm{N} / \mathrm{m}^{2}\right)$ is the material hardness of the softer material (Fig. 21). Micro-hardness have been done and $H=700 \mathrm{Hv}$ (MPa). In Eq.35, $K$ is a dimensionless coefficient characteristic of the materials in contact. Simple microscopic interpretation of the "Archard's factor" $K$ is frequently given (see Felder [12] or François \& al. [13] for example) which suggests a relation between the factor $K$ and the dynamic friction coefficient $\mu$. In that way, a low dynamic friction coefficient leads to very small wear because of slight dissipated power during slip coupled with a small wear factor. We will test this assumption in the following.

Tangential dissipated work is calculated by the product $T$ times $L$. In an instantaneous form of this model, the wear rate $\left(\mathrm{m}^{3} / \mathrm{s}\right)$ is directly proportional to the dissipated power $P_{d}$ as defined in Eq.36.

$\dot{W}=\frac{K}{H} P_{d}$

$P_{d}$ is not uniformly distributed over the contact area and it is necessary to calculate the tangential surface-traction distribution $\tau$ and the sliding velocity $w_{g}$ at each point on the contact area to specify the distribution of dissipated energy per unit surface. We define the wear depth rate $\dot{u}(\mathrm{~m} / \mathrm{s})$ by Eq.37.

$\dot{u}=\frac{d \dot{W}}{d S}=\frac{K}{H} \tau w$

During a single pass of the roller on the cam track, the increment of the wear depth is obtained by an integration over the time (t) of the wear depth rate from zero to $\Delta t=2 a(y) / V$ where $V$ is the rolling velocity and $2 a(y)$ is the length of the contact stripe at abscise $y$ as shown in Fig. 22. This yields to the incremental wear depth per roller passage $\delta u / \delta n$ given by Eq.38 where $P_{l}(y)$ is the dissipated power per unit length. 
$\frac{\delta u(y)}{\delta n}=\frac{K}{H V} P_{l}(y)$

$P_{l}(y)$ is calculated by integration over $\mathrm{x}$ (rolling direction) of tangential traction $\tau(x, y)$ times sliding velocity $w(x, y)$. Those quantities are inputs for the wear simulation software. For each step of the simulation, wear is calculated from the dissipated power per unit length with Eq.38. In this form, our wear model is a local form of Archard law as presented by Jendel [14], Telliskivi [15] and Goryacheva\&al.[16].

The new profile is determined. Local curvatures are updated and a new Fastsim calculation is carried out. The evolution of wear profile can be estimated step by step.

\subsubsection{Numerical details on the simulation}

Chart of Fig.23 resumes the successive steps needed to solve the wear evolution problem. A single iteration ' $i$ ' is equivalent to a single roller passage on the contact zone but since the incremental wear is very small, the same geometrical conditions may be kept during a large number of passages. This also helps to reduce the number of calculations by considering that the wear evolution is linear during $N$ passages of roller. Further we will present the simulation of the wear test managed on an apparatus where 3 rollers are disposed at $120^{\circ}$ (Fig. 24). The duration $T$ of the test is linked to the number of iterations $I$ and the rotation speed of the specimen $\omega_{r}$ by Eq.39.

$T=\frac{I N}{3 \omega_{r}}$

To reduce the number of iterations I, it is necessary to choose $N$ as big as possible. We propose to compare the length $L_{h}$ in the $y$ direction and depth $u_{\max }$ of the wear profile at the first iteration with the height $P_{g}$ of the roller profile for the same length (Fig. 25). The wear $u(y)$ at the first iteration will be given by Eq.40.

$$
u(y)=\frac{N K P_{l}(y)}{H V}
$$

When the dissipated power per unit length is symmetric, the wear depth is related to the maximum value of $P_{l}(y)$ by Eq.41.

$u_{\max }=\frac{N K P_{l 0}}{H V} \quad$ with $P_{l 0}=P_{l}(y=0)$

$y=0$ is the centre of the wear profile. If we assume that the roller profile is circular near the contact zone (see Fig.26) and $P_{g}$ is directly related to $L_{h}$. One defines the $D$ parameter as the ratio between $P_{g}$ and $u_{\max }$. 
$D=\frac{P_{g}}{u_{\max }}$

Combining Eqs.41 and 42, one can evaluate the number of passages $N$ in one iteration by Eq.43.

$N=\frac{H V P_{g}}{D K P_{l 0}}$

Several numerical simulations have been carried out and showed that the $D$ parameter must be greater than 1 to ensure simulation stability. Since $N$ must be as big as possible the practical value of $D=1.1$ is chosen to guarantee fast simulation and good stability.

Another source of instability is the sharp edges that appears at each iteration when the incremental wear is taken off the profile. On Fig.27 one can see the specimen profile after several roller passages. It can be subdivided into 3 zones: 2 external zones where profile is a straight line (no wear) and a central zone where we can make a parabolic interpolation: $z_{2}=a y^{2}+c$. In the two other zones, we have: $z_{1}=z_{3}=0$. Discontinuities of the first and second derivatives at junction between the three zones will cause fluctuation that becomes more and more numerous as number of iteration increases and calculation results have no signification. We smooth these sharp points by using an hyperbolic tangent function (see Eq.44) for continuity of the 3 zones.

$\psi(y)=\tanh \left(\alpha\left(y-y_{0}\right)\right)$

$\alpha$ and $y_{0}$ are parameters to fit for the best efficiency of the method. If we limit to one half of the profile, the Eq.45 gives the 'smoothen' equation from which one can compute the curvature needed for the SHAD and Fastsim calculations.

$z(y)=\frac{1}{2}\left((1-\psi(y)) z_{1}(y)+(1+\psi(y)) z_{2}(y)\right)$

Practically $y_{0}$ must be close from the abscissa where region separates and $\alpha$ must be small enough so that the angle between profiles $z_{1}$ and $z_{2}$ is not to much 'smoothed'.

\subsubsection{Example: wear during a rolling test}

In this last section, we use the approaches presented above to simulate a non Hertzian case of wear. The case is representative of a rolling test where the 2 bodies - a roller and a cylindrical specimen - are revolution bodies (Fig.2). Definition of the problem can be done by the profile of the roller and the evolution of the profile of the specimen from one iteration to another. The numerical values that have been chosen for this example have been 
identified on friction and wear tests: $\mu=0.08, K=0.01, \omega_{\mathrm{r}}=950 \mathrm{rpm}$. Longitudinal creepage is evaluated to $v_{x}=$ $0.065 \%$, and transversal and spin creepages are supposed to be null: $v_{y}=\phi=0, F=300 \mathrm{~N}$. Figure 28 , shows the evolution of the maximum wear $u_{\max }$ versus cycles: it shows a non linear phase in the beginning (from 0 to 0.5 $10^{6}$ cycles) and after that wear evolution is linear. Following the wear profile during the simulation one can explain this partition by the evolution of the contact during iterations: at first contact is quasi-hertzian, then wear modifies progressively the curvatures of the specimen profile. This phase, describing the accommodation between contacting bodies, is known as the "running-in" stage. At the end of this phase (about 0.5 million cycles), contact becomes conform (see Fig.29). Load and geometry remains identical and the wear evolution becomes linear versus cycle. Final wear profile is identical to the roller profile and this is confirmed experimentally on section made from the specimen.

\section{Conclusions}

Simplified approaches for non Hertzian rolling contact problems have been developed for wear simulation. First, a simplified approach called SAHD aims to find not only the best contact area between two solids in contact but also the best pressure distribution in a short CPU time. It is based on the Hertz theory and its ability to solve critical problems such as conforming contact and contact with negative curvature has been proved. Coupled to a diffusion method, information is diffused between stripes and irregularities coming from the discontinuities of curvatures correction are smoothed. An optimal value of the diffusion coefficient is given and it has been shown that it gives good results for a wide range of normal loads and curvatures.

Second, a simplified approach based on the Fastsim algorithm is chosen to solve the tangential problem in order to evaluate dissipated energy in the contact and finally wear evolution. Coupled with the Archard's law, wear profiles can be actualised at each step of the simulation and the evolution of wear versus time can be followed. Difficulties arising from the curvature discontinuities introduced by the actualisation of the profile have been treated by the use of a 'smoothing' function and a D parameter has been introduced to accelerate the 
simulation by calculating the maximum number of roller passages to be considered in a single iteration.

Further studies are running actually as a prolongation of the presented work. Extension to non stationary wear processes by taking into account the evolution of the normal load, the curvatures and other parameters versus time has been presented in [17] and has direct applications to industrial machinery. Taking into account the experimental dispersions during wear tests and the dispersion of the wear profiles once the test is finished, one must treat the identification (see [18] for details) of the wear law coefficient $K$ as a stochastic inversion problem. This work has been initiated in [19] and must be completed by taking into account model errors due to the simplified approaches used instead of the exact theory. It is possible to evaluate the viability of the solution by a non parametric probabilistic approach as the one developed by Soize and al. [20]. This is the next step of the work to be done.

\section{References}

[1] L. Chevalier, H. Chollet, Endommagement des pistes de roulement, Mec. Ind. 1 (2000) 77-103.

[2] L. Chevalier, S. Cloupet, C. Soize, Probabilistic approach for wear modelling in steady state rolling contact, Wear 258 (2005) 1543-1554.

[3] J.J. Kalker, B. Jacobson, Rolling Contact Phenomena: Linear Elasticity, Berlin, 2000, p.84.

[4] J. B. Ayasse, H.Chollet, Determination of the Wheel Rail Contact Patch in Semi-Hertzian Conditions, Veh. Syst. Dyn., 43 3, (2005) 161-172.

[5] B. Nayroles, G. Touzot, P. Villon, La méthode des éléments diffus, C. R. Acad. Sci. Paris, Ser. II 313 (1991) $133-138$

[6] J. J. Kalker, A Fast Algorithm for the Simplified Theory of Rolling Contact, Vehicle Syst. Dyn. 11 (1982) 113.

[7] L. Chevalier, S. Cloupet, A. Eddhahak-Ouni, Contributions à la modélisation simplifiée de la mécanique des contacts roulants, Mec. Ind, 7 (2006) 155-168

[8] J.J.Kalker, The contact between wheel and rail, Delft: Delft University of Technology, Report of the Department of mathematics and informatics, 82 27, (1982) 1-36.

[9] J.J. Kalker, Wheel/rail wear calculations with the program CONTACT, Contact Mechanics and Wear of Rail/Wheel System, 2 (1987), 3-26.

[10] J.F. Archard, Contact and rubbing of flat surfaces, J. Appl. Phys., 24 (1953) 981-988.

[11] Zi-Li Li, J.J Kalker, Simulation of Severe Wheel-Rail Wear, Proceeding International Conference Computers in Railways, ed. WITPRESS, Southampton UK, 6 (1998) 393-402.

[12] E.Felder, Mécanismes physiques et modélisation mécanique du frottement entre corps solides, Mec. Ind. 1, 6 (2000) 555-561.

[13] D.François, A.Pineau, A.Zaoui, Comportement mécanique des matériaux, Tome 2, Hermès, 1993, p. 401450 and p. 159-166.

[14] T. Jendel, Prediction of wheel profile wear-comparisons with field measurements, Wear, 253 (2002) 89-99.

[15] T. Telliskivi, U. Olofsson, Wheel-rail wear simulation, Wear. 257 (2004) 1145-1153.

[16] I.G. Goryacheva, P.T. Rajeev, T.N. Farris, Wear in Partial Slip Contact, Journal of Tribology, 123 (2001) 848-856. 
[17] A. Eddhahak-Ouni, Simulation de l'usure superficielle dans les contacts roulants : mise en place des méthodes adaptées aux cas non stationnaires, Thesis, Université de Marne la Vallée (2006).

[18] L. Chevalier, S. Cloupet, M. Quillien, Friction and Wear during a bi-disc test under severe conditions, Trib. Inter. 39, (2006). 1376-1387.

[19] S. Cloupet, Simulation de l'usure superficielle par microglissement dans les contacts roulants came - galet : approche probabiliste des dispersions expérimentales, Thesis, Ecole Normale Supérieure de Cachan (2006).

[20] C. Soize, Random Matrix Theory for Modelling Uncertainties in Computational Mechanics, Comp. Meth. In App. Mech. 194, 12-16 (2005) 1333-1366. 


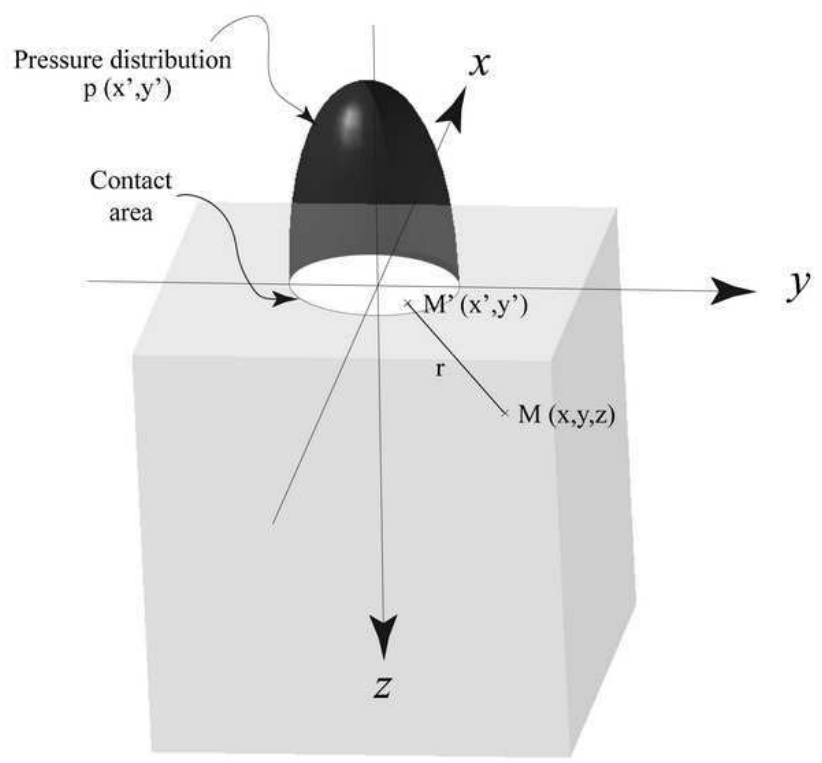

Fig. 1. Position of points $\mathrm{M}$ and M' 

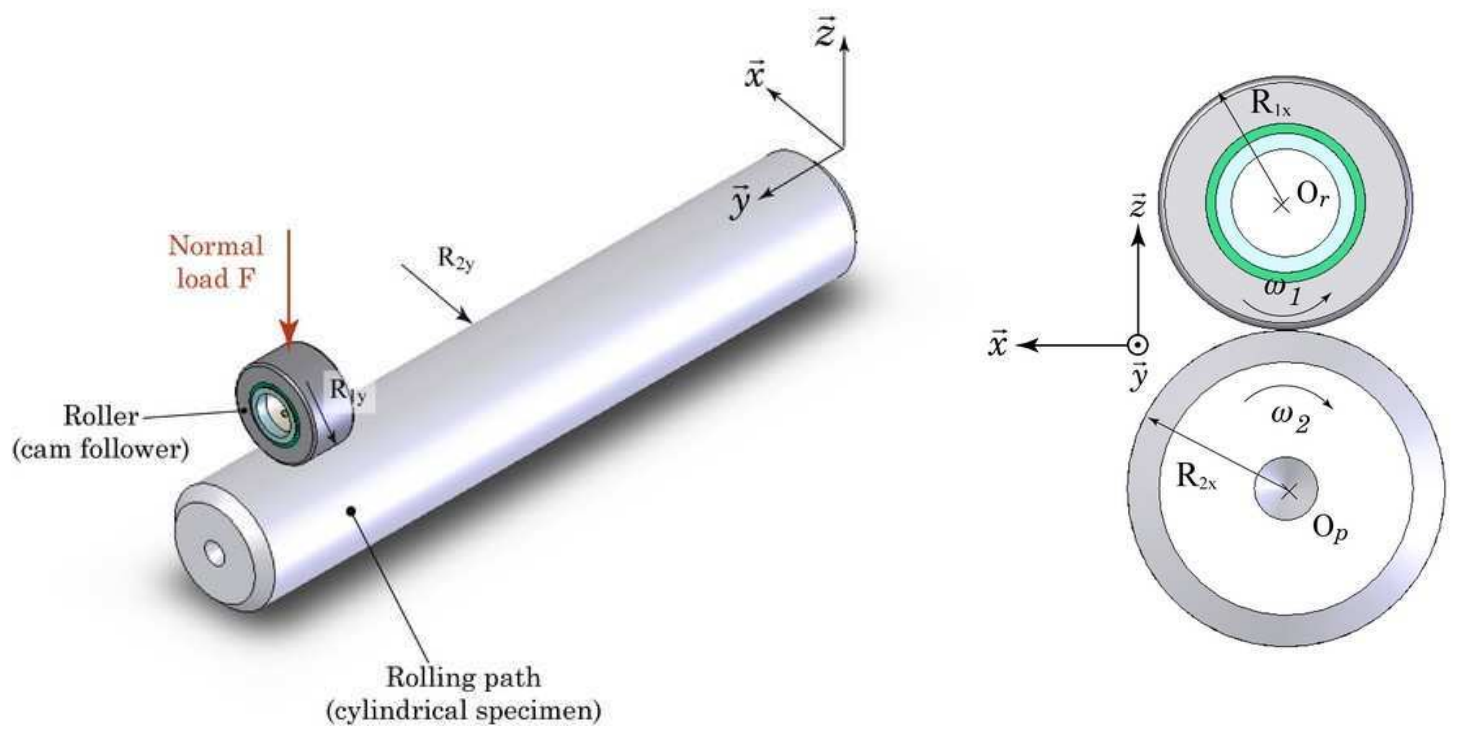

Fig. 2. Position of the two solids in the Cartesian coordinate system. 


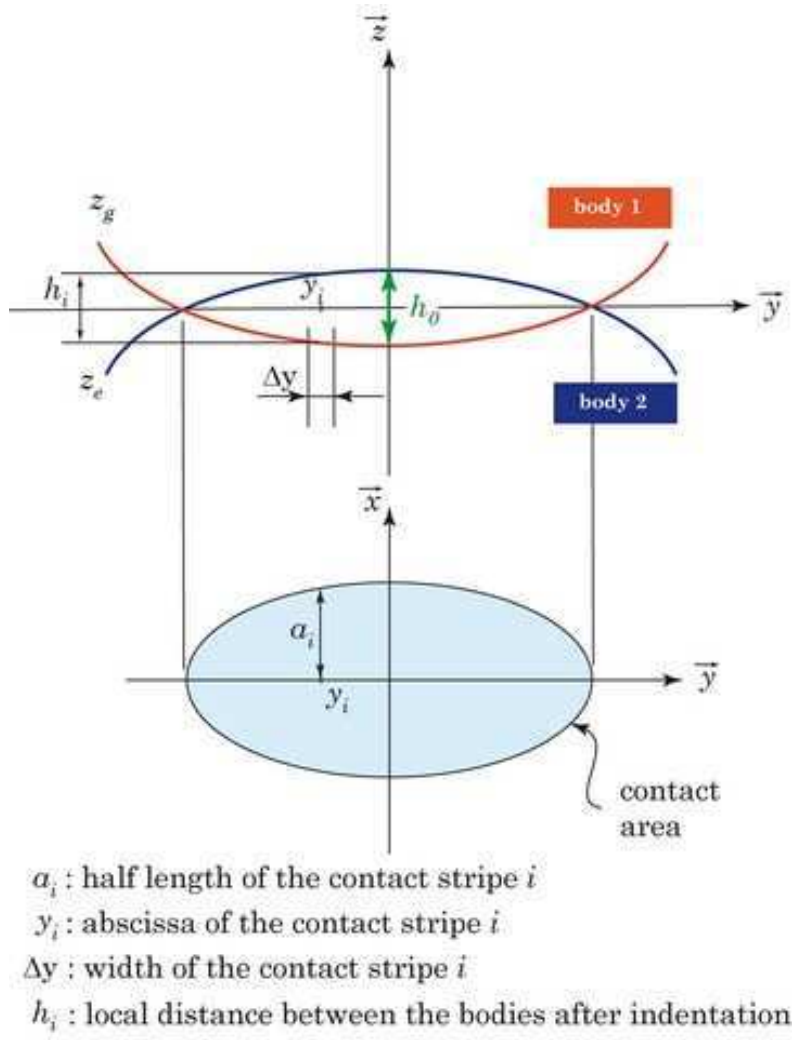

Fig. 3. Definition of the geometrical characteristics of the indentation method. 


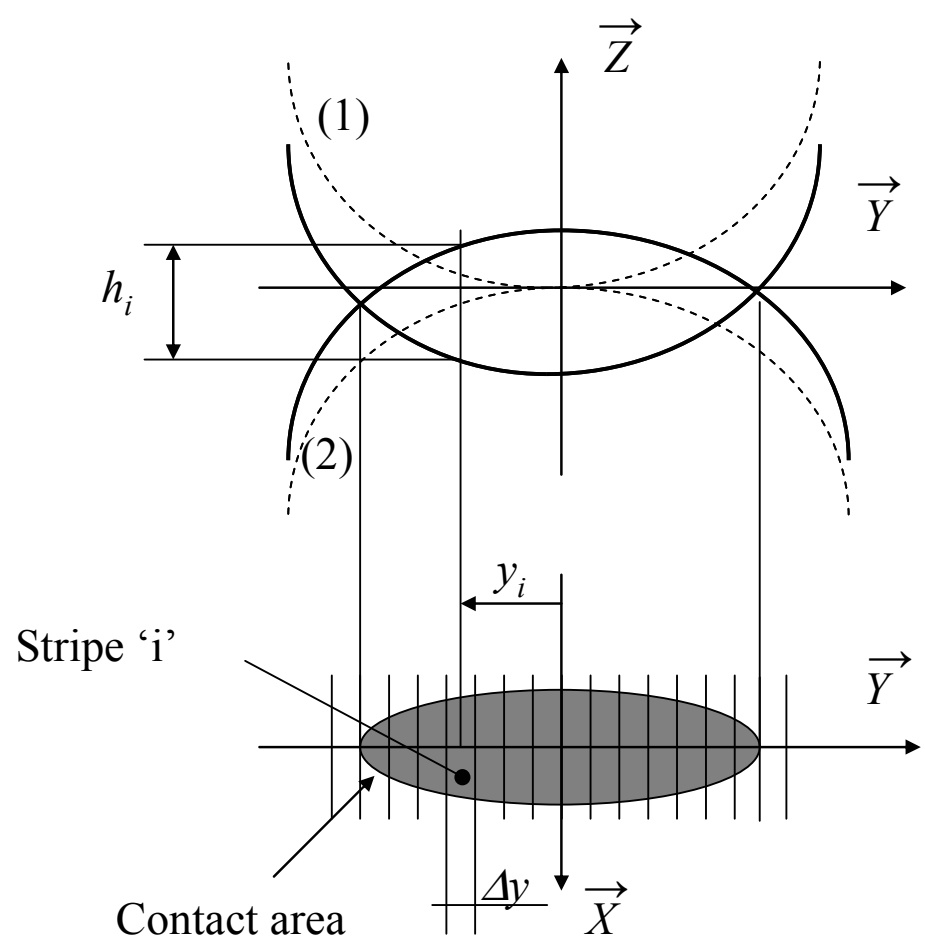

Fig.4: stripes definition 


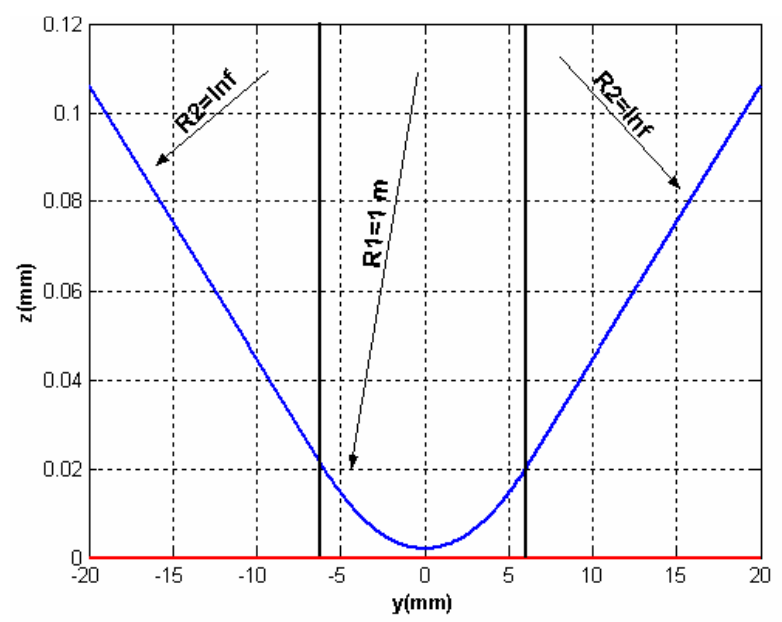

Fig. 5. Symmetric profile of the roller along $y$. 


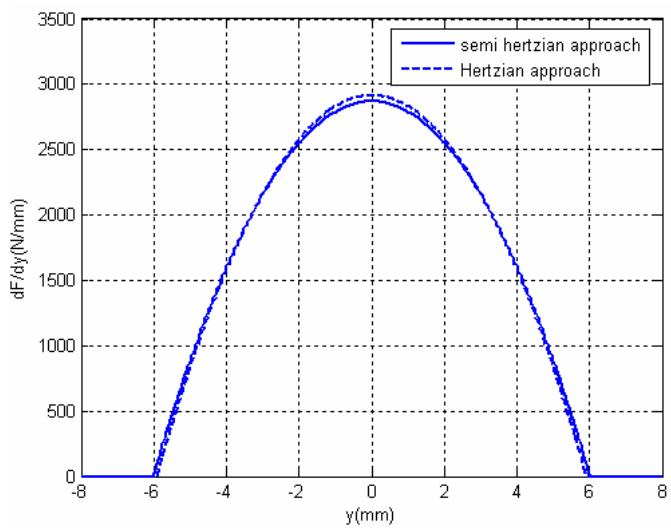

(a)

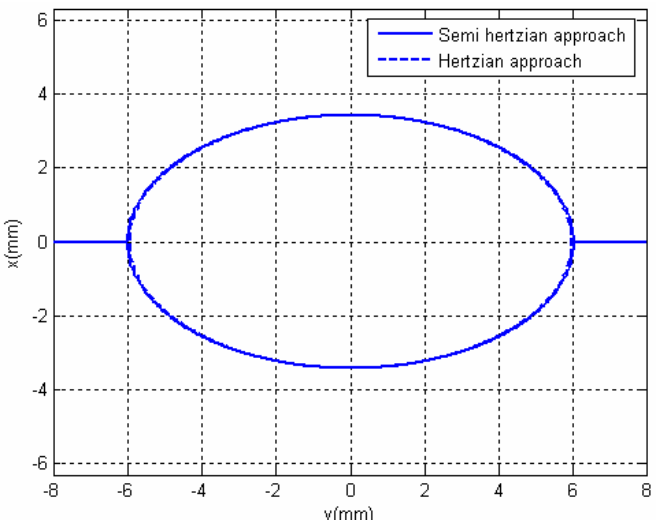

(b)

Fig. 6. (a), (b) Respectively contact patch and distribution of the normal load by unit of length by Semi Hertzian method and analytical solution. 


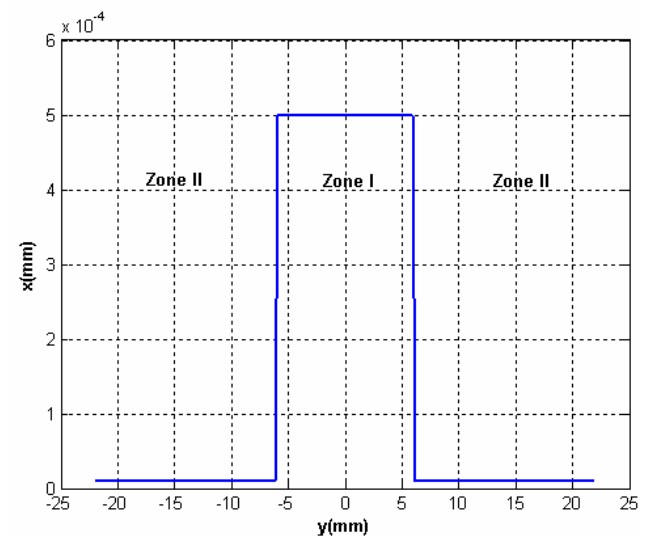

(a)

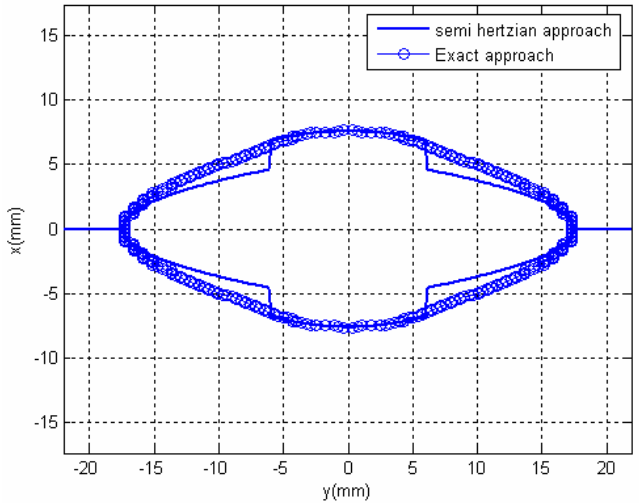

(b)

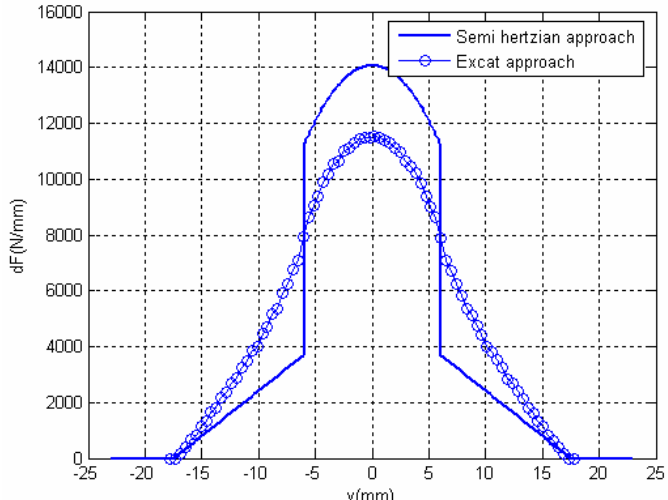

(c)

Fig. 7. (a) Curvature $B$ along $y$, (b) Contact areas obtained by exact and Semi Hertzian methods, (c) Normal load by length obtained by exact and Semi Hertzian methods 


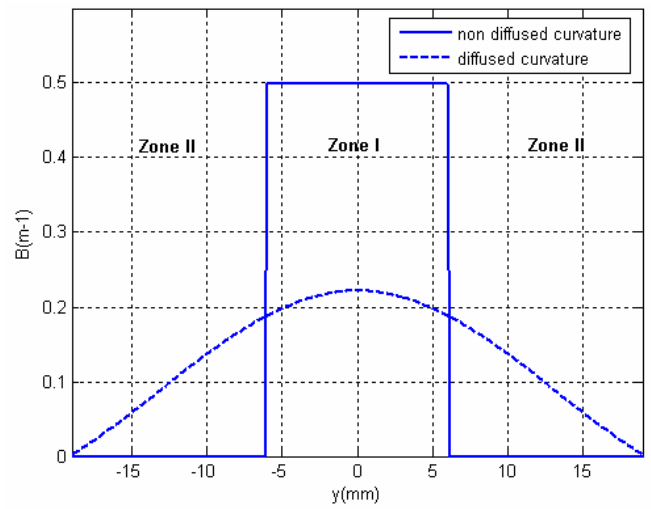

(a)

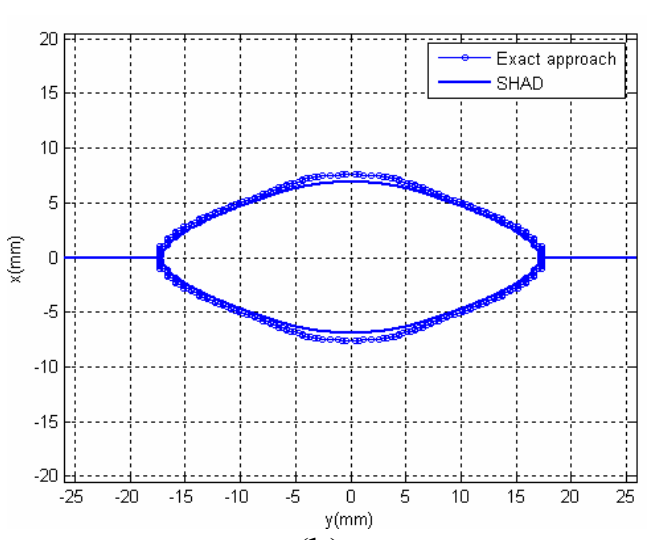

(b)

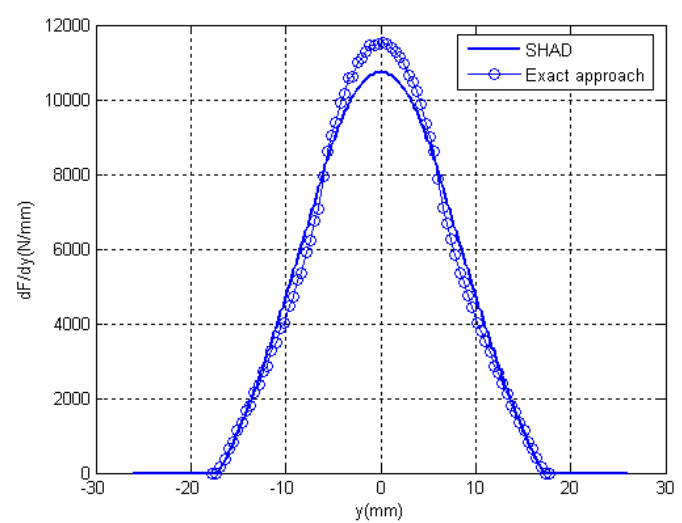

(c)

Fig. 8. (a) Curvature $B$ along $y$ before and after diffusion, (b) Contact areas obtained by

SHAD and exact method, (c) Normal load by length obtained by SHAD and exact method 

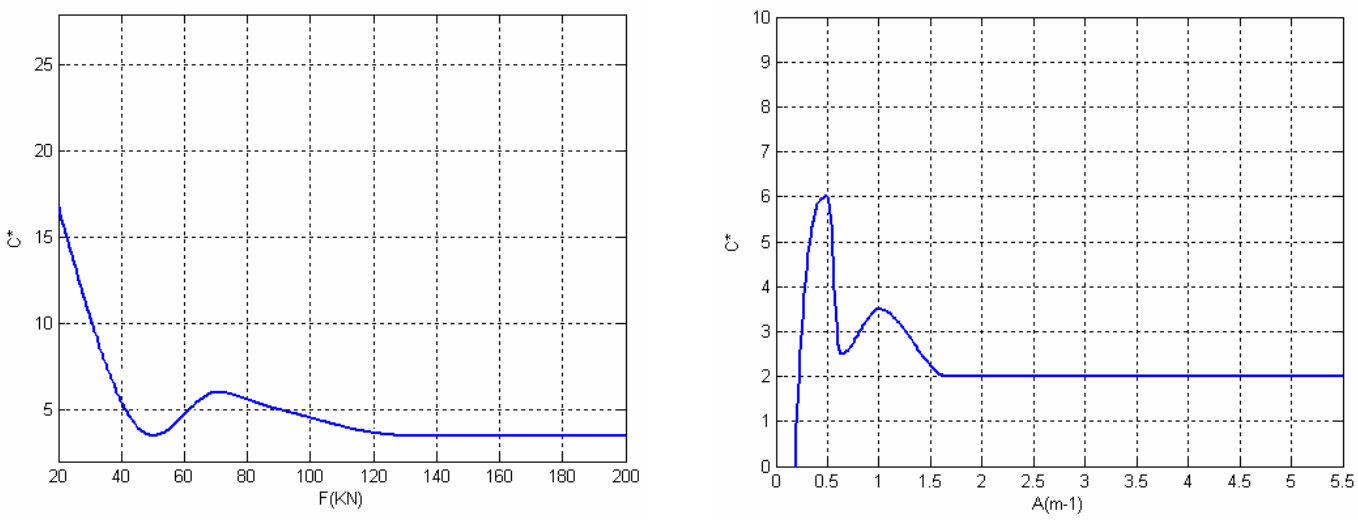

Fig. 9. Variation of $C^{*}$ with normal load (left) and with the curvature $A$ (right). 


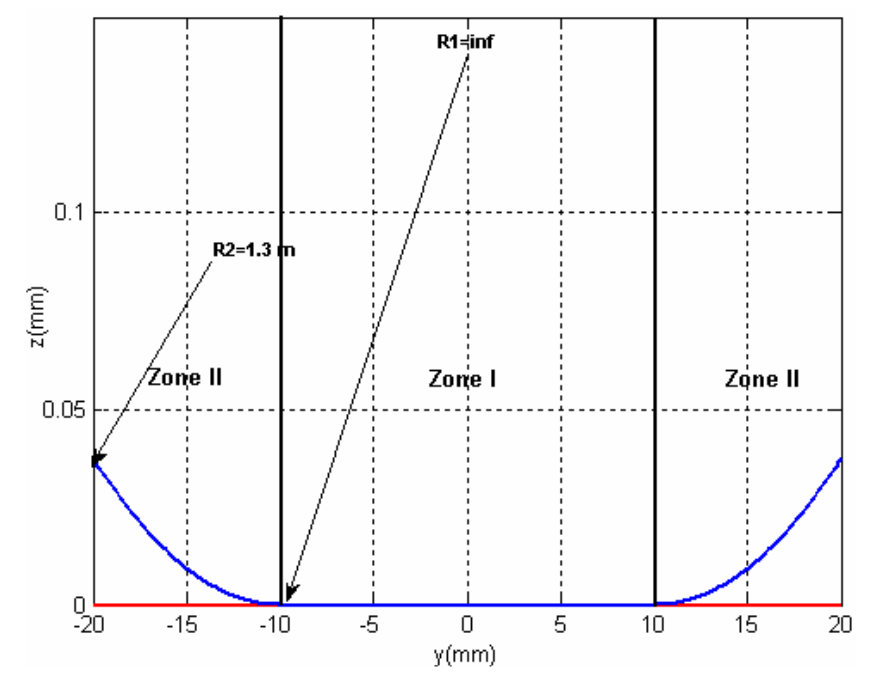

Fig.10. Profile of the roller in the plane $(y O z)$. 


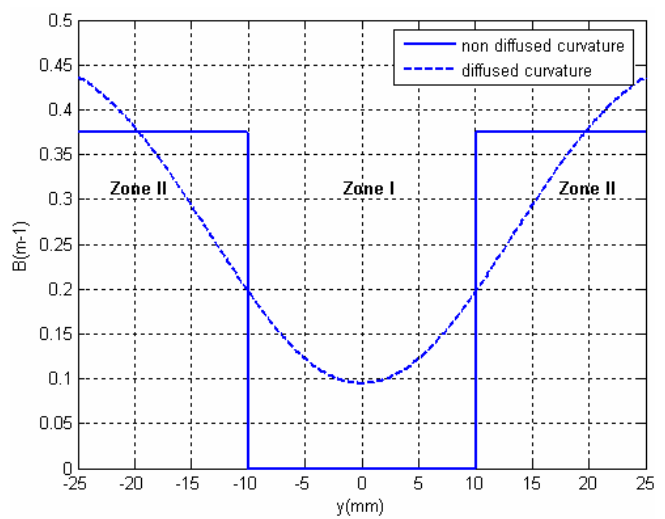

(a)

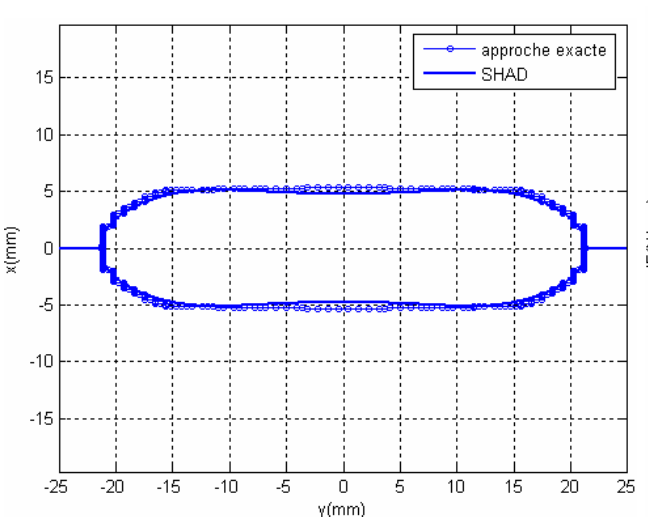

(b)

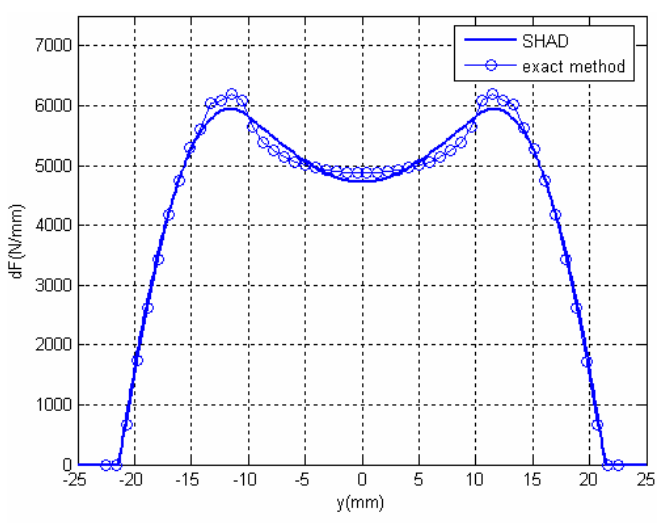

(c)

Fig. 11. (a) Curvature $B$ along $y$ before and after diffusion, (b) Contact areas obtained by SHAD and exact method, (c) Normal load by length obtained by SHAD and exact method 


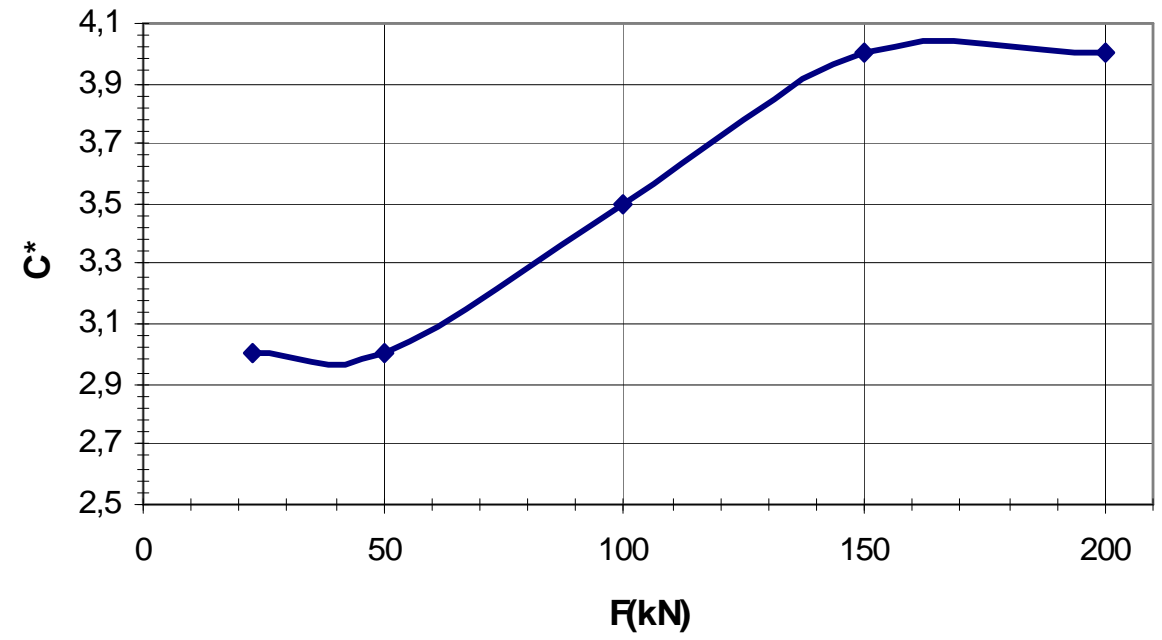

Fig. 12. Variation of $C^{*}$ with normal load. 


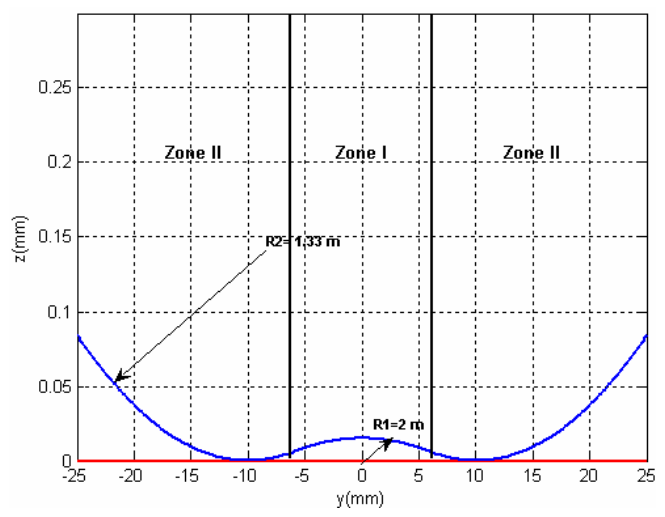

Fig. 13. Roller's profile in the plane (yoz). 


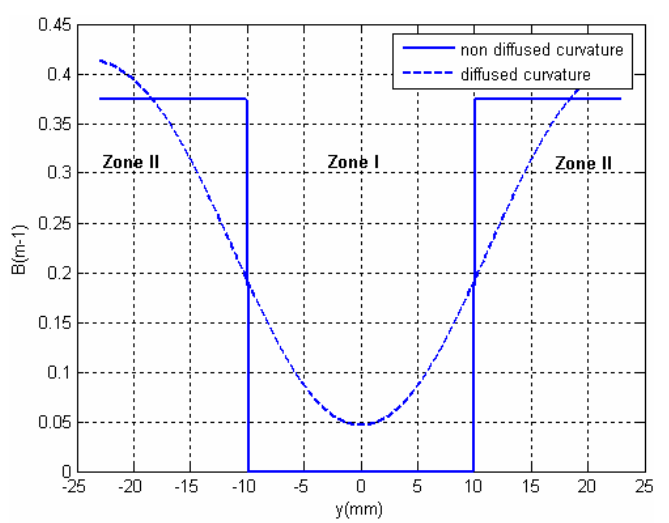

(a)

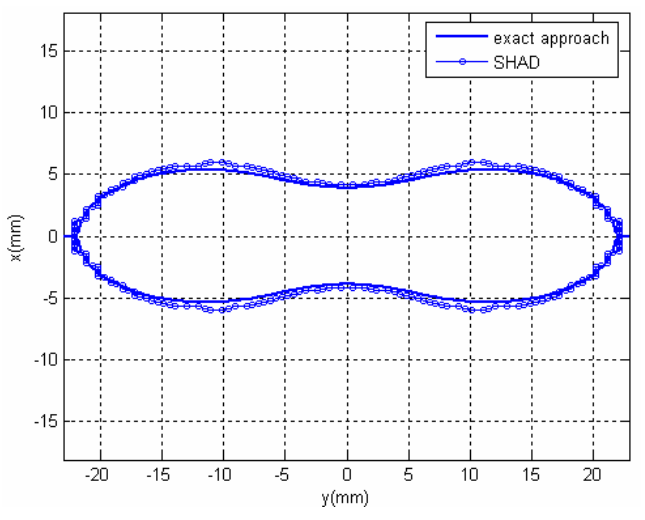

(b)

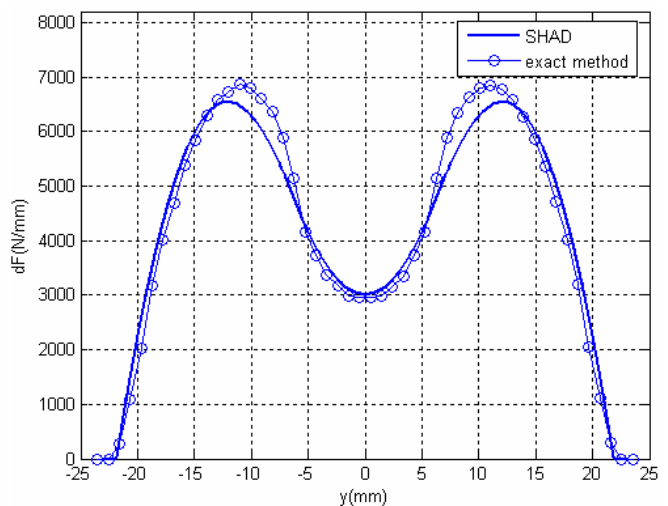

(c)

Fig. 14. (a) Curvature $B$ along $y$ before and after diffusion, (b) Contact areas obtained by SHAD and exact method, (c) Normal load by length obtained by SHAD and exact method 


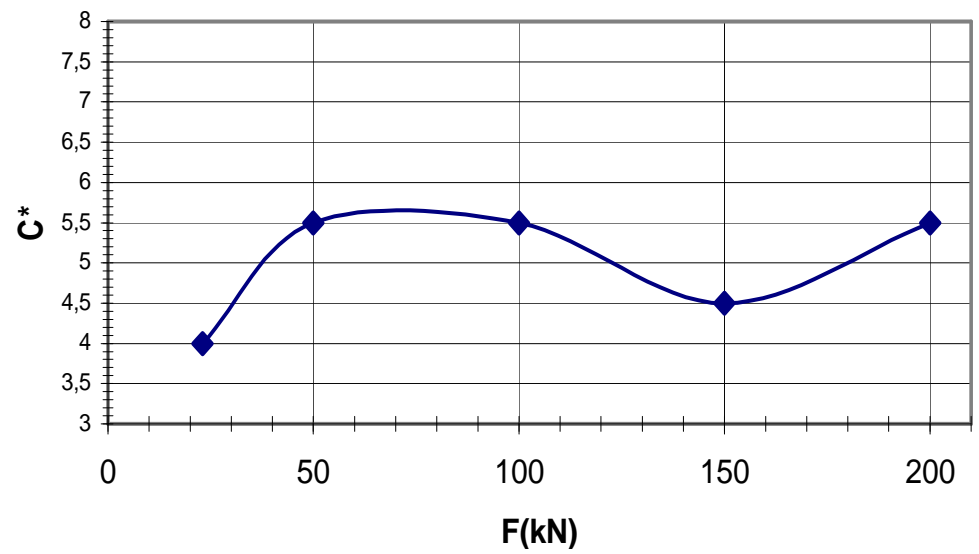

Fig. 15. Variation of $C^{*}$ with normal load. 


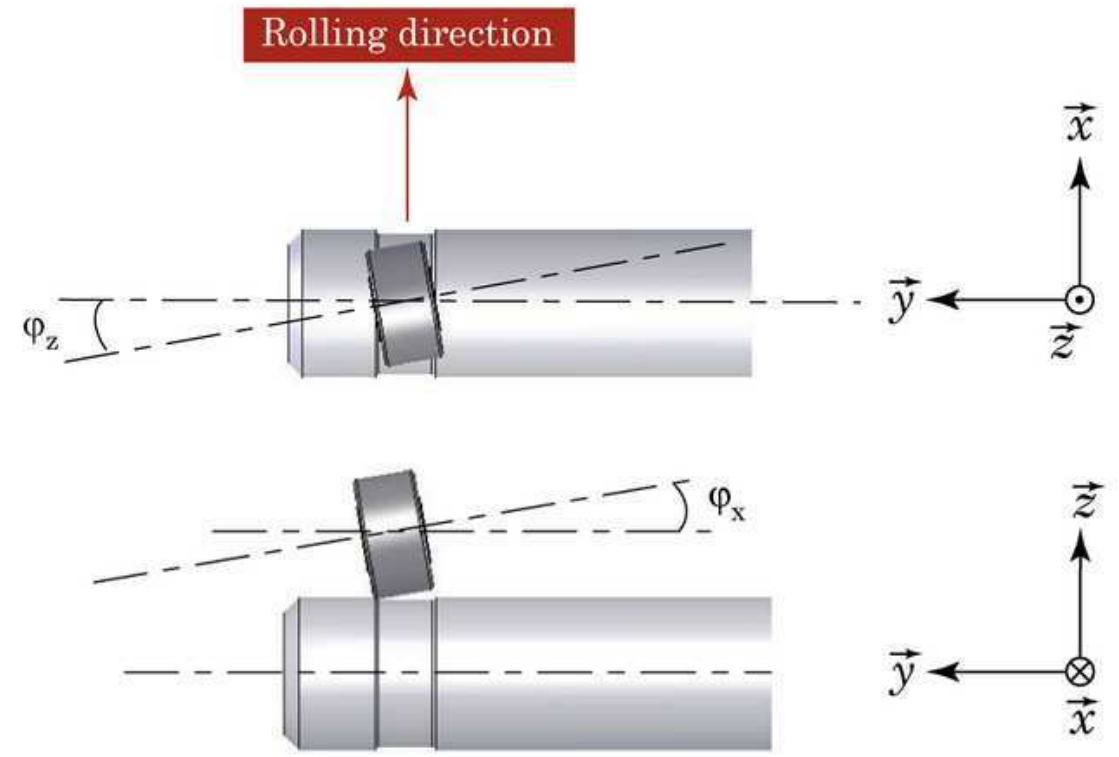

Fig. 16. Definitions of yaw angle $\varphi_{\mathrm{z}}$ and spin angle $\varphi_{\mathrm{x}}$. 


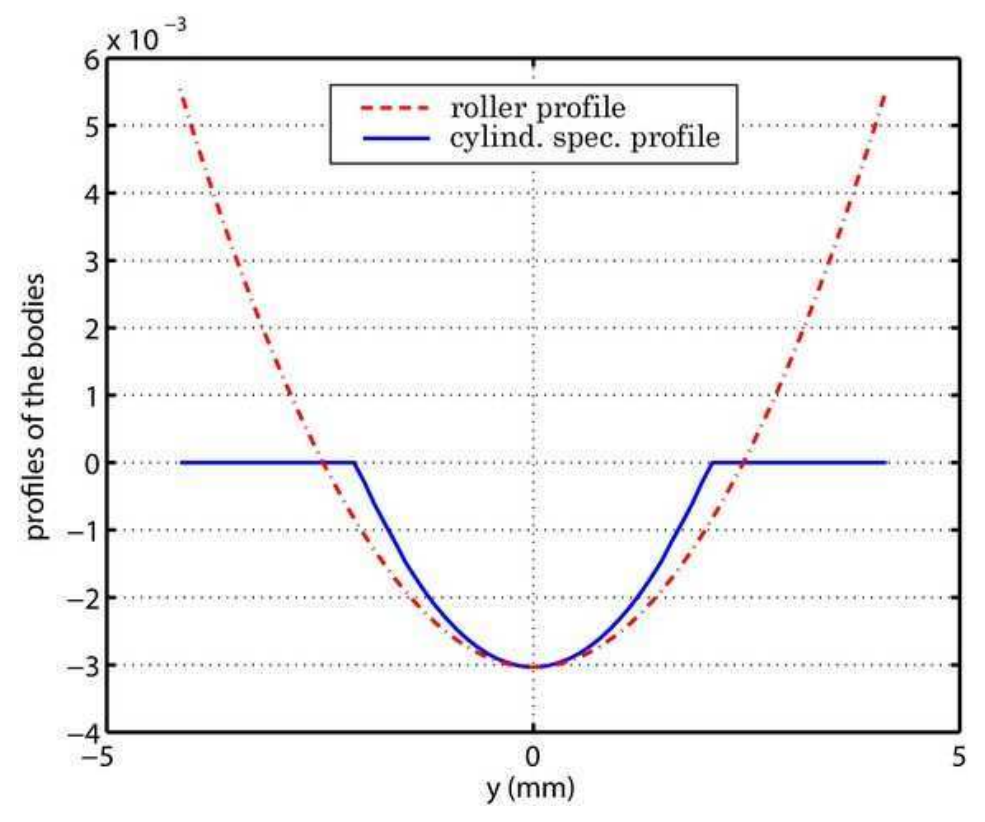

Fig. 17. Test case (benchmark) of non-hertzian contact. 

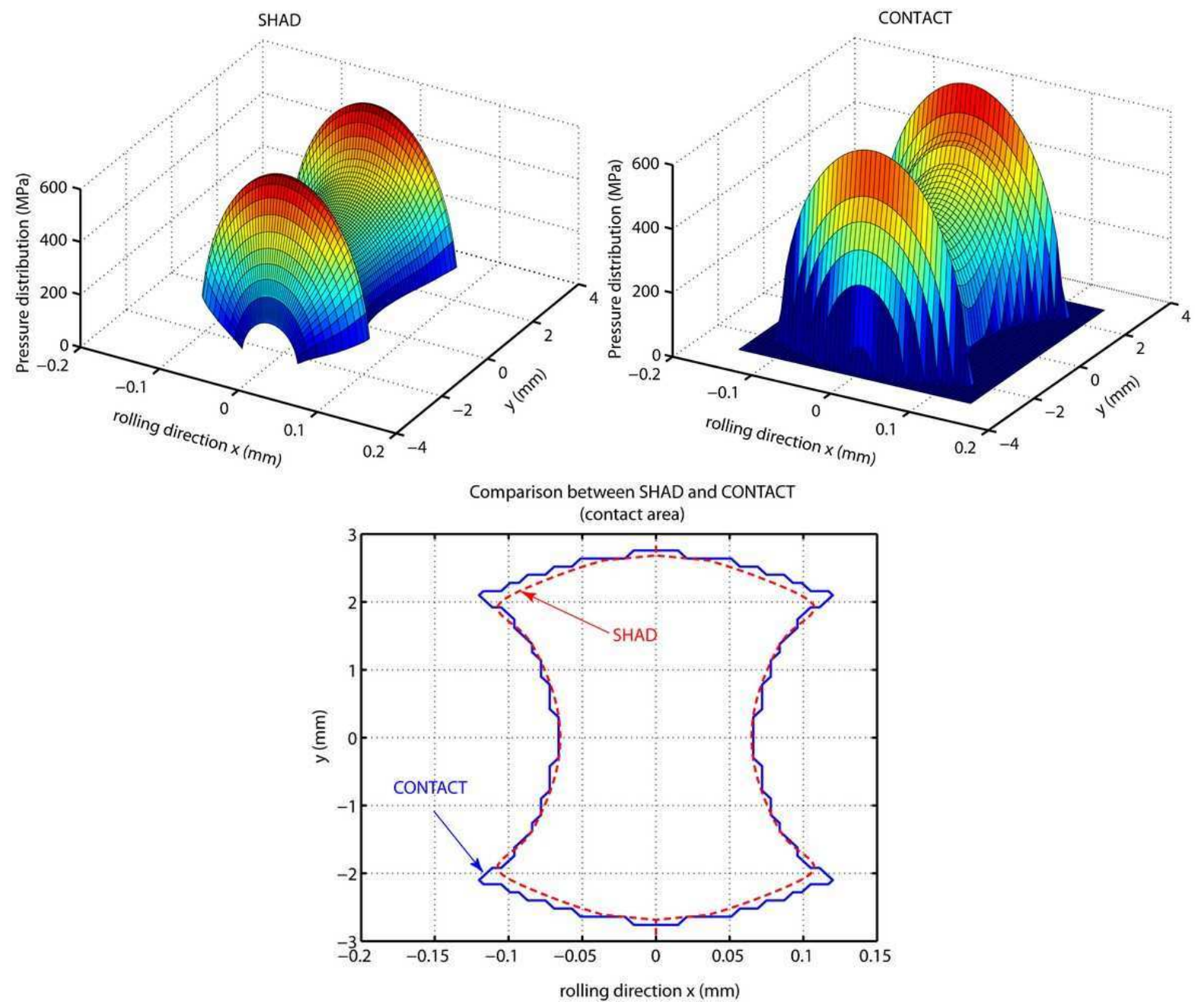

Fig. 18. Comparison between CONTACT and SHAD results of normal problem. 

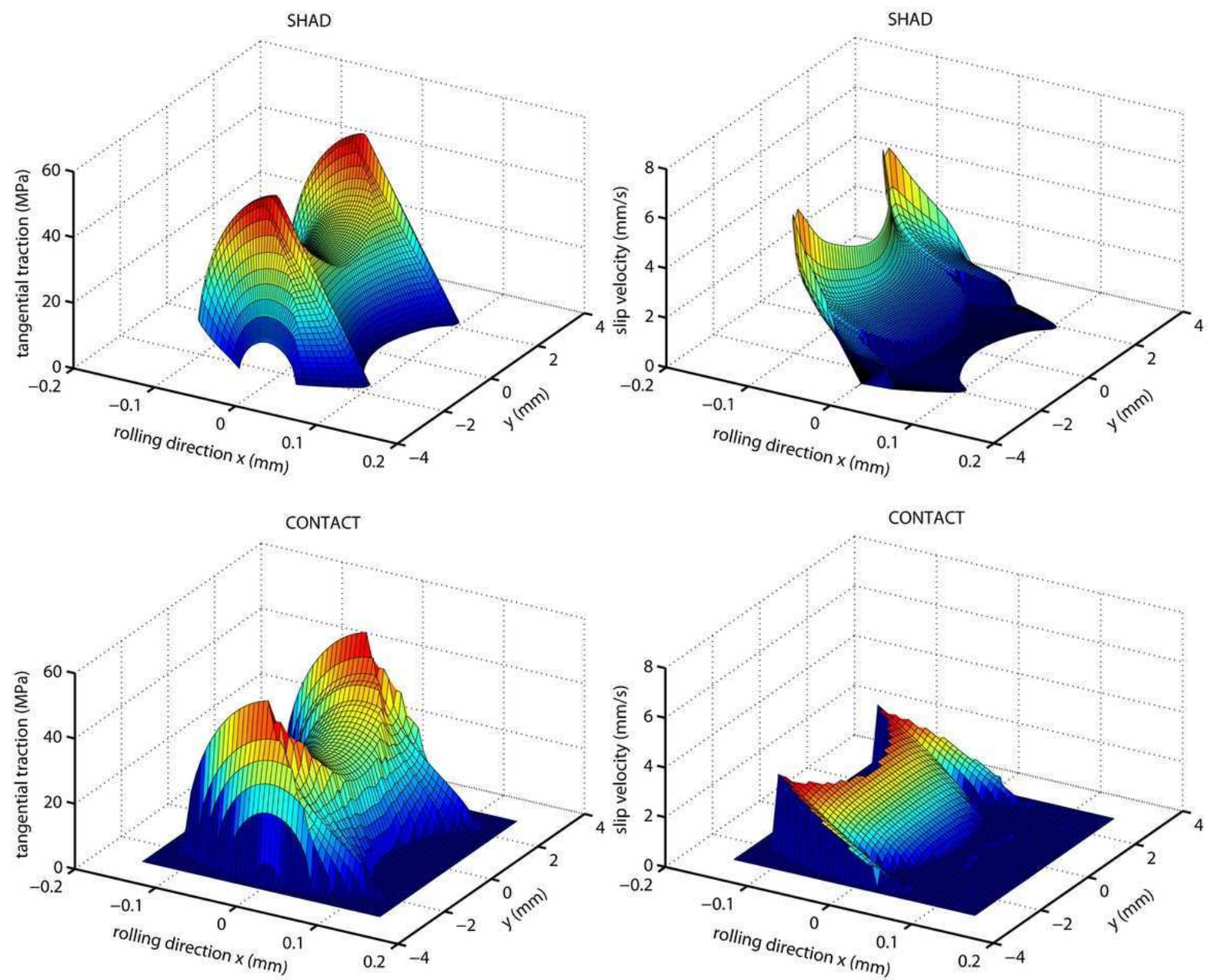

Fig. 19. Comparison between CONTACT and SHAD results of tangential problem. . Shear force distribution and slip velocity distribution results 


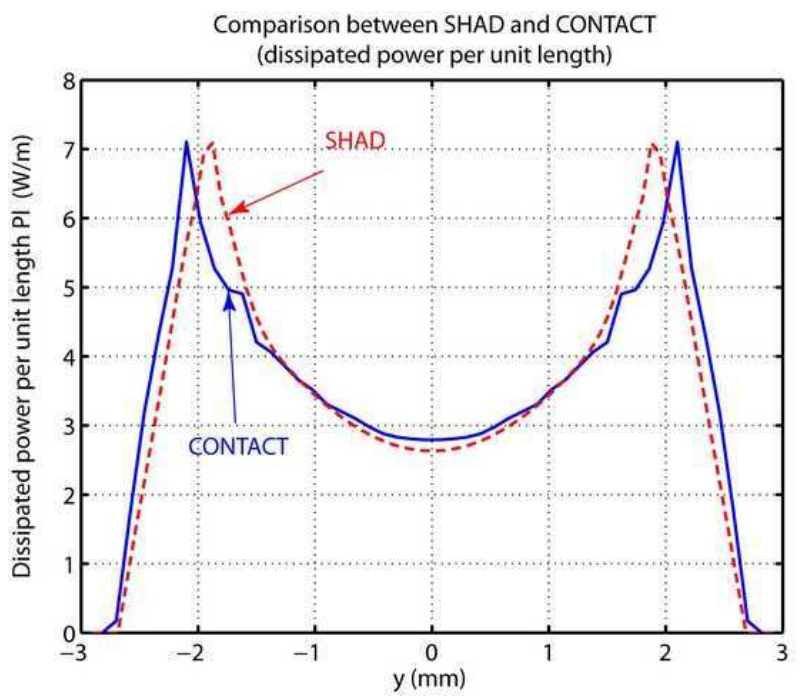

Fig. 20. Dissipated power per unit length $P_{1}$ obtained with CONTACT and SHAD 


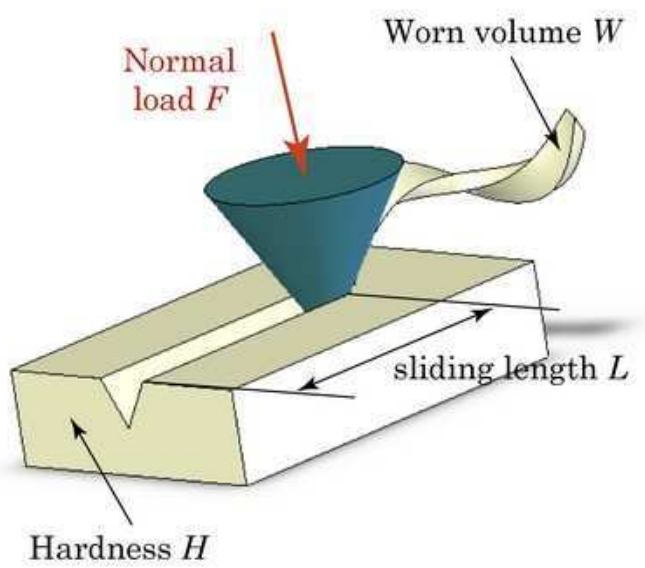

Fig. 21. Definition of Archard's wear law 

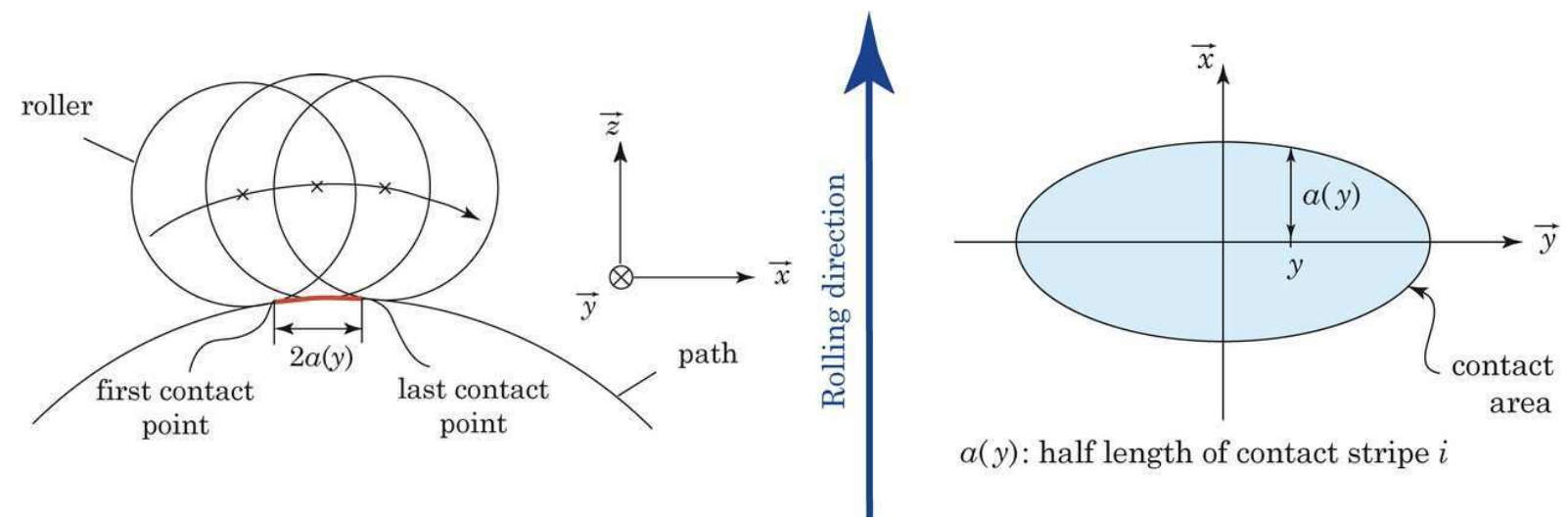

$a(y)$ : half length of contact stripe $i$

Fig.22. Half length of the contact stripe $a(y)$ and contact area S 


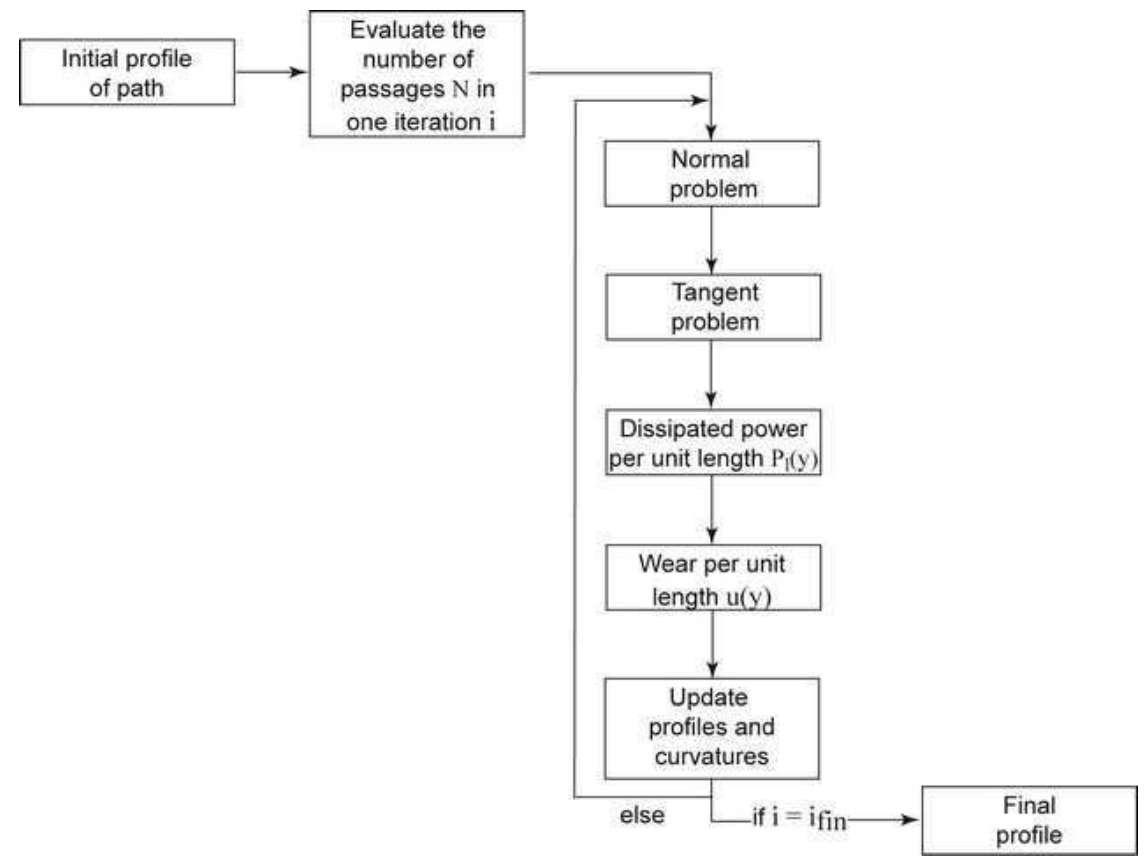

Fig. 23. Incremental procedure to solve the wear problem. 


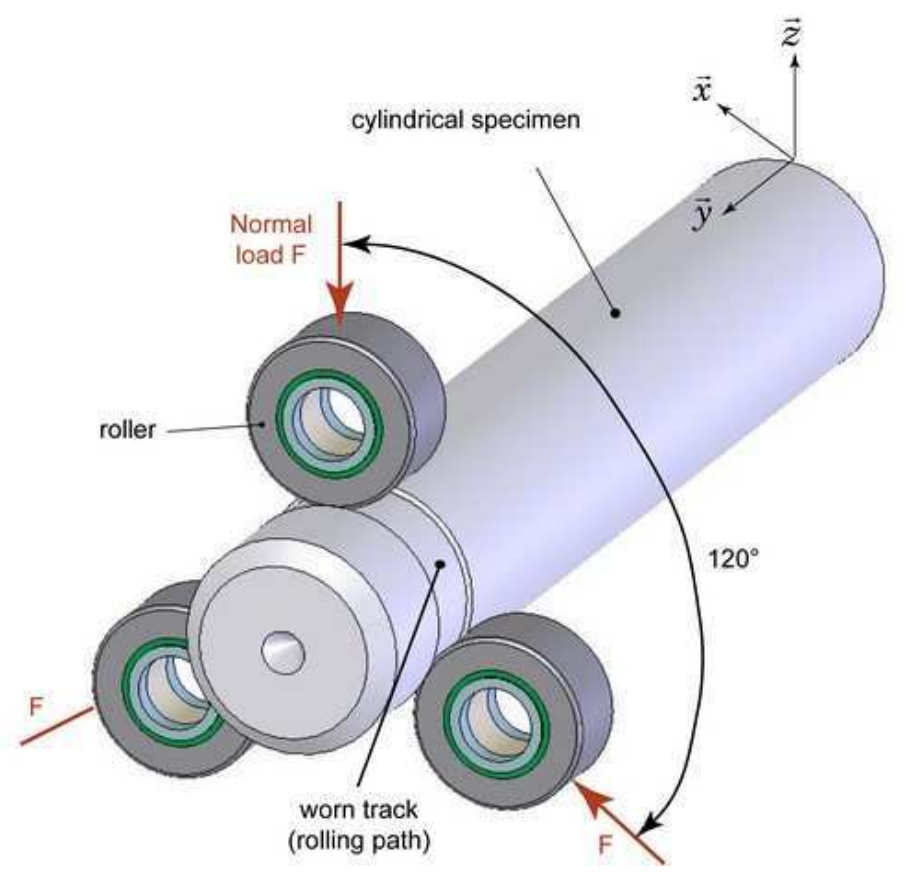

Fig. 24. Test bench. 


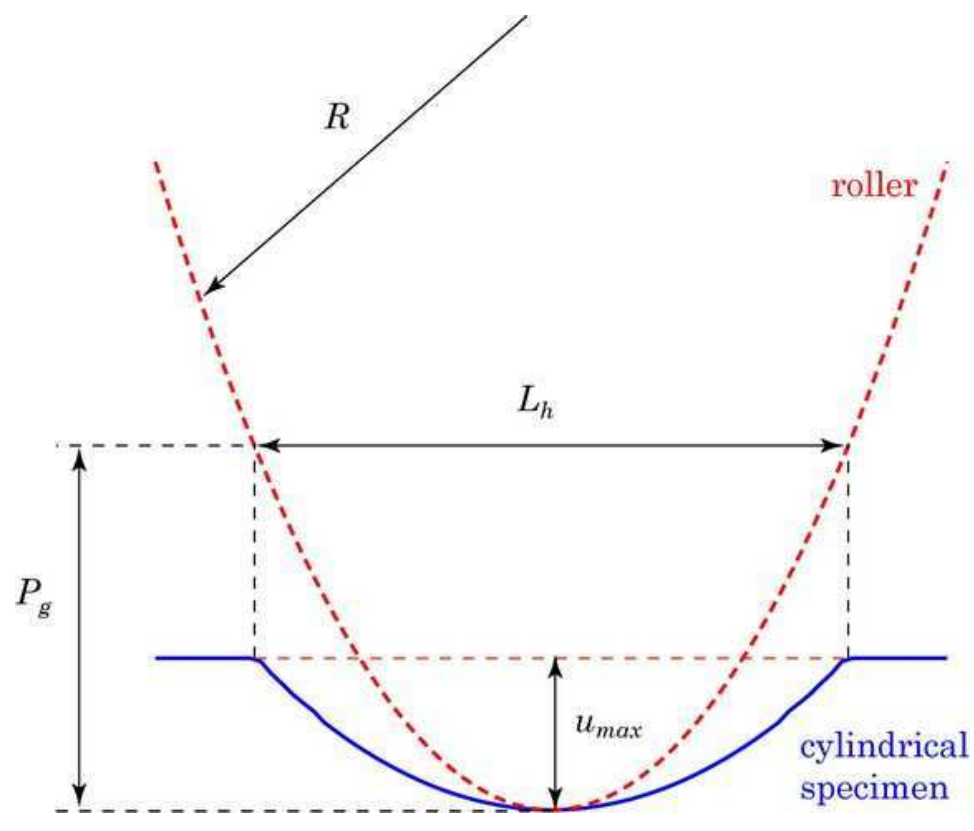

Fig. 25. Definition of $D$ parameter. 


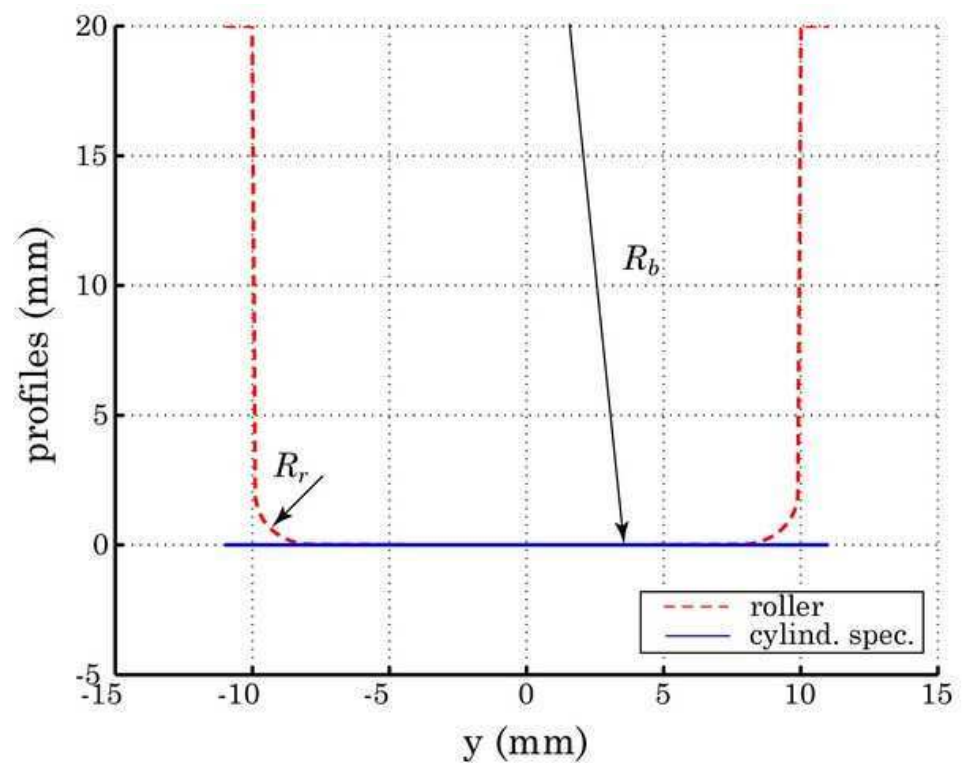

Fig. 26. Definition of initials profiles of roller and cylindrical test (specimen). 


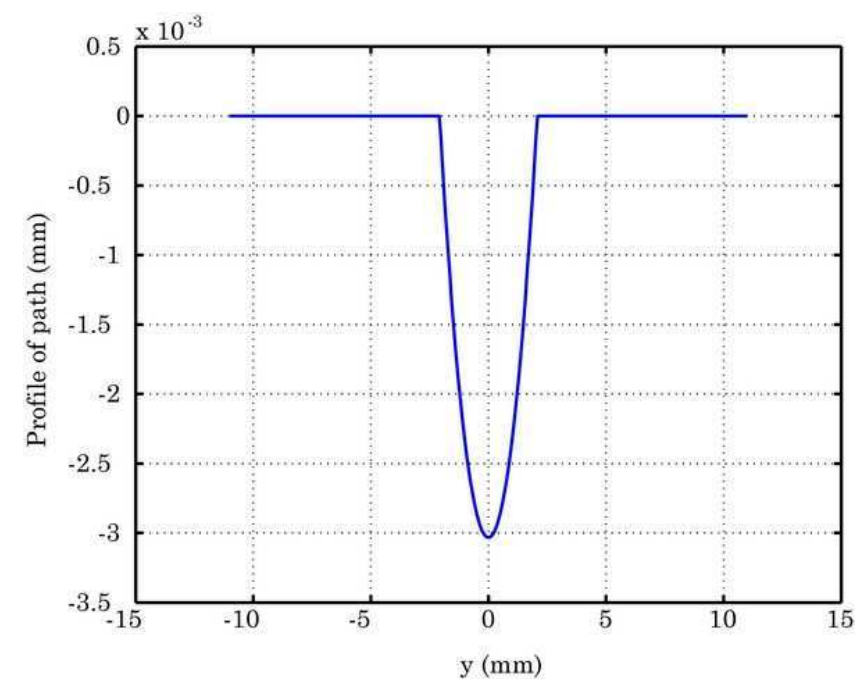

Fig. 27. Wear profile of specimen after several roller passages. 


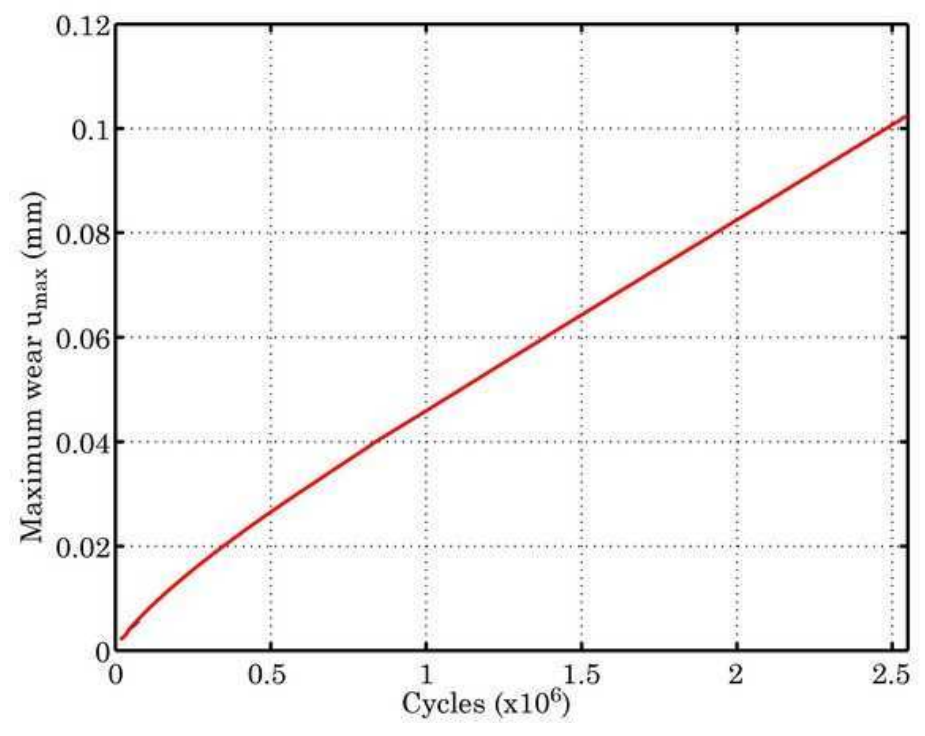

Fig. 28. Evolution of the maximum wear $u_{\max }$ versus cycles. 

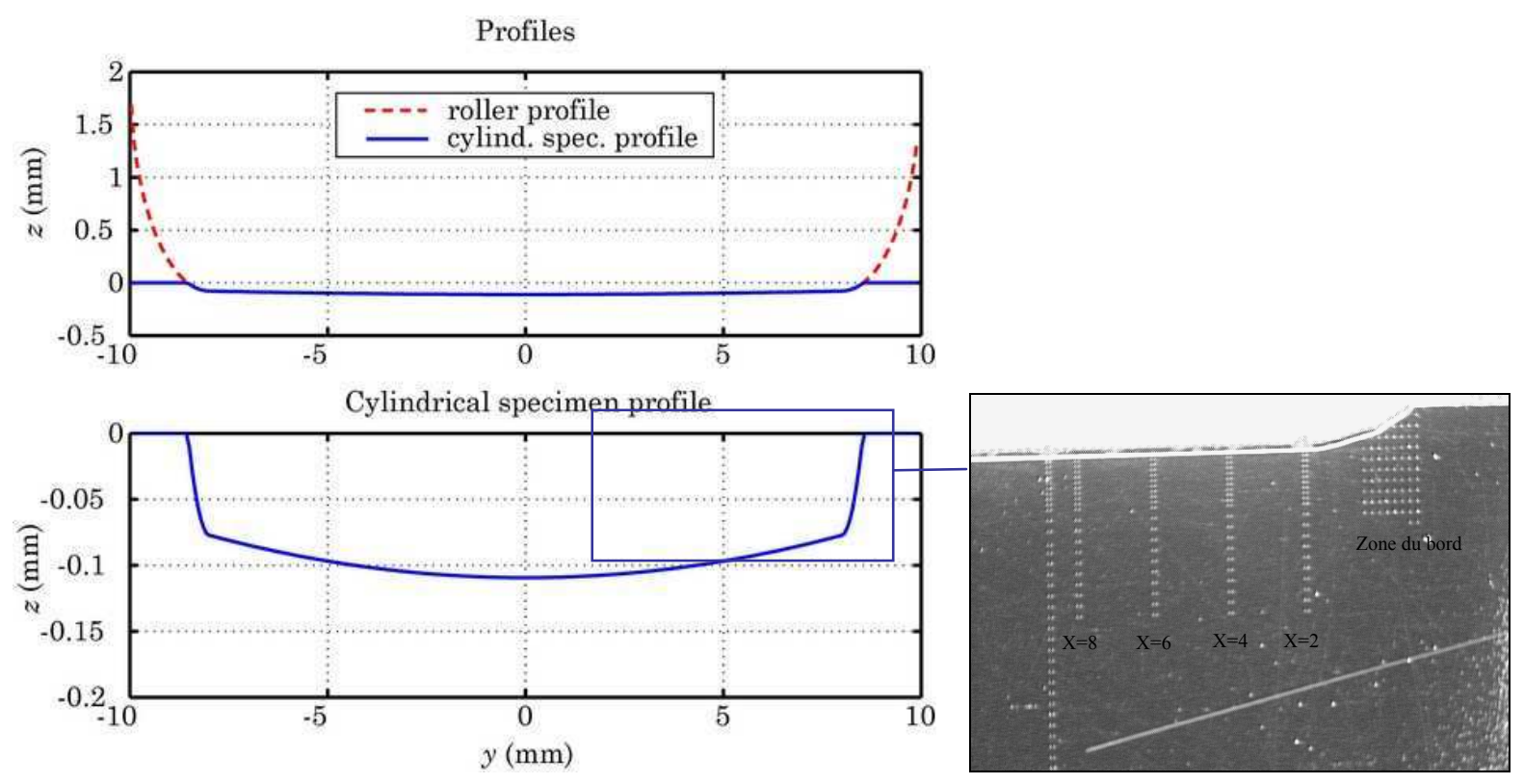

Fig. 29. Specimen profile at the end of the simulation (left) and comparison with wear profile measured. 